\title{
PARITY IN PARTITION IDENTITIES
}

\author{
GEORGE E. ANDREWS
}

\begin{abstract}
This paper considers a variety of parity questions connected with classical partition identities of Euler, Rogers, Ramanujan and Gordon. We begin by restricting the partitions in the Rogers-Ramanujan-Gordon identities to those wherein even parts appear an even number of times. We then take up questions involving sequences of alternating parity in the parts of partitions. This latter study leads to: (1) a bi-basic $q$-binomial theorem and $q$-binomial series, (2) a new interpretation of the Rogers-Ramanujan identities, and (3) a new natural interpretation of the fifth-order mock theta functions $f_{0}(q)$ along with a new proof of the Hecke-type series representation.
\end{abstract}

\section{INTRODUCTION}

Parity has played a role in partition identities from the beginning. For example [7; p. 5]

Euler's Partition Identity. The number of partitions of any positive integer $n$ into distinct parts equals the number of partitions of $n$ into odd parts.

Equivalently in terms of generating functions: for $|q|<1$, [7; p. 5, eq. (1.2.5)]

$$
\prod_{n=1}^{\infty}\left(1+q^{n}\right)=\prod_{n=1}^{\infty} \frac{1}{1-q^{2 n-1}}
$$

Now the Rogers-Ramanujan identities [7; p. 109] do not immediately involve parity.

First Rogers-Ramanujan Identity. The number of partitions of any positive integer $n$ into distinct non-consecutive parts equals the number of partitions of $n$ into parts congruent to $\pm 1(\bmod 5)$.

Equivalently in terms of generating functions: for $|q|<1$, [7; p. 113, Cor. 7.9]

$$
1+\sum_{n=1}^{\infty} \frac{q^{n^{2}}}{(1-q)\left(1-q^{2}\right) \cdots\left(1-q^{n}\right)}=\prod_{n=1}^{\infty} \frac{1}{\left(1-q^{5 n-1}\right)\left(1-q^{5 n-4}\right)} .
$$

However B. Gordon [15], [17] and H. Göllnitz [13], [14] independently introduced parity considerations as follows:

First Göllnitz-Gordon Identity. The number of partitions of $n$ into distinct non-consecutive parts with no even parts differing by exactly 2 equals the number of partitions of $n$ into parts $\equiv 1,4$, or $7(\bmod 8)$.

2000 Mathematics Subject Classification. Primary 11P83, 11P81, 05A19, 05A17.

Key words and phrases. partitions, Rogers-Ramanujan, parity index.

Partially supported by National Science Foundation Grant DMS 0200097. 
Equivalently in terms of generating functions: for $|q|<1$,

$$
1+\sum_{n=1}^{\infty} \frac{q^{n^{2}}(1+q)\left(1+q^{3}\right) \cdots\left(1+q^{2 n-1}\right)}{\left(1-q^{2}\right)\left(1-q^{4}\right) \cdots\left(1-q^{2 n}\right)}=\prod_{n=1}^{\infty} \frac{1}{\left(1-q^{8 n-1}\right)\left(1-q^{8 n-4}\right)\left(1-q^{8 n-7}\right)}
$$

There are several results of this sort related to the Rogers-Ramanujan identities [14]. In addition, in his Lost Notebook [8], [10] Ramanujan found $q$ series identities such as $\left[8 ;\right.$ p. 57 , eq. $\left.(1.10)_{R}\right]$

$$
\begin{aligned}
& \sum_{n=1}^{\infty} \frac{\left(1-q^{n}\right)}{\left(1+q^{n}\right)}\left(1+\sum_{m=1}^{\infty} \frac{q^{m(m+1) / 2}}{\left(1-q^{2}\right)\left(1-q^{4}\right) \cdots\left(1-q^{2 m}\right)}\right)+ \\
& \sum_{n=0}^{\infty} \frac{(-1)^{n} q^{n(n+1) / 2}}{(1+q)^{2}\left(1+q^{2}\right)^{2} \cdots\left(1+q^{n}\right)^{2}}=2 \sum_{n=0}^{\infty} \frac{(-q)^{n(n+1) / 2}}{\left(1+q^{2}\right)\left(1+q^{4}\right) \cdots\left(1+q^{2 n}\right)} .
\end{aligned}
$$

It was noted in [5; p. 55], that

$$
\sum_{n=0}^{\infty} O E(n) q^{n}:=1+\sum_{n=1}^{\infty} \frac{q^{n(n+1) / 2}}{\left(1-q^{2}\right)\left(1-q^{4}\right) \cdots\left(1-q^{2} n\right)}
$$

is the generating function for $O E(n)$, the number of partitions of $n$ into distinct parts in which the parity of parts alternates and the smallest part is odd.

These examples from the literature foreshadow the deeper examination of parity in partition identities that will be undertaken here.

Our first major exploration will concern parity in the celebrated Rogers-Ramanujan-Gordon identities [1], [16]:

Rogers-Ramanujan-Gordon identities. Let $B_{k, a}(n)$ denote the number of partitions of $n$ for the form

$$
b_{1}+b_{2}+\cdots+b_{j},
$$

where $b_{i} \geqq b_{j+1}, b_{i}-b_{i+k-1} \geqq 2$ and at most $a-1$ of the $b_{i}$ are equal to 1 and $1 \leqq a \leqq k$. Let $A_{k, a}(n)$ denote the number of partitions of $n$ into parts $\not \equiv 0, \pm a$ $(\bmod 2 k+1)$. Then for all $n \geqq 0$,

$$
A_{k, a}(n)=B_{k, a}(n)
$$

After Gordon's proof of this theorem in 1961 [16], there was subsequently discovered a generating function version in 1974 [6]: for $|q|<1$

$$
\sum_{n_{1}, \ldots, n_{k-1} \geqq 0} \frac{q^{N_{1}^{2}+N_{2}^{2}+\cdots+N_{k-1}^{2}+N_{a}+N_{a+1}+\cdots+N_{k-1}}}{(q ; q)_{n_{1}}(q ; q)_{n_{2}} \cdots(q ; q)_{n_{k-1}}}=\prod_{\substack{n=1 \\ n \neq 0, \pm a(\bmod 2 k+1)}}^{\infty} \frac{1}{1-q^{n}}
$$

where

$$
N_{j}=n_{j}+n_{j+1}+\cdots+n_{k-1}
$$

and

$$
(A ; q)_{n}=(A)_{n}=(1-A)(1-A q) \cdots\left(1-A q^{n-1}\right) .
$$

We now involve parity restrictions:

Theorem 1. Suppose $k \geqq a \geqq 1$ are integers with $k \equiv a(\bmod 2)$. Let $\mathcal{W}_{k, a}(n)$ denote the number of those partitions enumerated by $B_{k, a}(n)$ with the added restriction that even parts appear an even number of times. If $k$ and $a$ are both even, let $G_{k, a}(n)$ denote the number of partitions of $n$ in which no odd part is repeated and no even part $\equiv 0, \pm a(\bmod 2 k+2)$. If $k$ and a are both odd, let $G_{k, a}(n)$ denote 
the number of partitions of $n$ into parts that are neither $\equiv 2(\bmod 4)$ nor $\equiv 0, \pm a$ $(\bmod 2 k+2)$. Then for all $n \geqq 0$,

$$
\mathcal{W}_{k, a}(n)=G_{k, a}(n) .
$$

Theorem 2. Suppose $k \geqq a \geqq 1$ with $k$ odd and a even. Let $\bar{W}_{k, a}(n)$ denote the number of those partitions enumerated by $B_{k, a}(n)$ with added restriction that odd parts appear an even number of times. Then

$$
\sum_{n \geqq 0} \bar{W}_{k, a}(n) q^{n}=\prod_{m=1}^{\infty} \frac{1}{1+q^{2 m-1}} \prod_{\substack{n=1 \\ n \neq 0, \pm a(\bmod 2 k+2)}}^{\infty} \frac{1}{1-q^{n}} .
$$

In analogy with (1.4), we shall prove equivalent generating function identities.

Theorem 3. For $k \geqq a \geqq 1, k \equiv a(\bmod 2)$,

$$
\begin{aligned}
\sum_{n_{1}, n_{2}, \ldots, n_{k-1}} & \frac{q^{N_{1}^{2}+N_{2}^{2}+\cdots+N_{k-1}^{2}+2 N_{a}+2 N_{a+2}+\ldots 2 N_{k-2}}}{\left(q^{2} ; q^{2}\right)_{n_{1}}\left(q^{2} ; q^{2}\right)_{n_{2}} \cdots\left(q^{2} ; q^{2}\right)_{n_{k-1}}} \\
= & \sum_{n \geqq 0} \mathcal{W}_{k, a}(n) q^{n} \\
= & \sum_{n \geqq 0} G_{k, a}(n) q^{n} \\
= & \frac{\left(-q ; q^{2}\right)_{\infty}\left(q^{a} ; q^{2 k+2}\right)_{\infty}\left(q^{2 k+2-a} ; q^{2 k+2}\right)_{\infty}\left(q^{2 k+2} ; q^{2 k+2}\right)_{\infty}}{\left(q^{2} ; q^{2}\right)_{\infty}}
\end{aligned}
$$

and if $k \geqq a \geqq 2, k$ odd, a even, then

$$
\begin{aligned}
\sum_{n_{1}, \ldots, n_{k-1} \geqq 0} \frac{q^{N_{1}^{2}+\cdots+N_{k-1}^{2}+n_{1}+n_{3}+\cdots+n_{a-3}+N_{a-1}+N_{a}+\cdots+N_{k-1}}}{\left(q^{2} ; q^{2}\right)_{n_{1}}\left(q^{2} ; q^{2}\right)_{n_{2}} \cdots\left(q^{2} ; q^{2}\right)_{n_{k-1}}} \\
\quad=\frac{\left(-q^{2} ; q^{2}\right)_{\infty}\left(q^{a} ; q^{2 k+2}\right)_{\infty}\left(q^{2 k+2-a} ; q^{2 k+2}\right)_{\infty}\left(q^{2 k+2} ; q^{2 k+2}\right)_{\infty}}{\left(q^{2} ; q^{2}\right)_{\infty}} \\
\quad=\frac{\left(q^{a} ; q^{2 k+2}\right)_{\infty}\left(q^{2 k+2-a} ; q^{2 k+2}\right)_{\infty}\left(q^{2 k+2} ; q^{2 k+2}\right)_{\infty}}{\left(-q ; q^{2}\right)_{\infty}(q ; q)_{\infty}} .
\end{aligned}
$$

The second part of our investigation concerns the alternating parity of parts related to partition functions like $O E(n)$ from (1.5). There will be numerous results derived on this topic. We sample the flavor of these results with the following unexpected variation on the first Rogers-Ramanujan identity. To do this we require the following definition:

The upper even index of a partition $\lambda_{1}+\lambda_{2}+\cdots+\lambda_{s}$ is the number of terms in the longest decreasing subsequence of parts $\lambda_{i}$ beginning with an even part and alternating in parity.

Corollary 15. The number of partitions of $n$ into distinct parts each larger than upper even index equals the number of partitions of $n$ into parts $\equiv \pm 1(\bmod 5)$.

Beyond this variation on the first Rogers-Ramanujan identity, we are led naturally to Ramanujan's mock theta functions [9].

Let us define RUE partitions of $n$ to be those described in the first part of Corollary 15. 
Theorem 17. Let $\mathrm{ru}_{e}(n)$ (resp. $\mathrm{ru}_{o}(n)$ ) denote the number of RUE partitions of $n$ with an even (resp. odd) upper even index. Then for the fifth order mock theta function $f_{0}(q)$, we have

$$
f_{0}(q):=\sum_{n=0}^{\infty} \frac{q^{n^{2}}}{(-q ; q)_{n}}=\sum_{n=0}^{\infty}\left(\mathrm{ru}_{e}(n)-\mathrm{ru}_{o}(n)\right) q^{n} .
$$

We shall, in addition, look at a variety of alternating parity questions, and shall be led inexorably to improving the development of the Hecke type series for $f_{0}(q)$. Surprisingly there is a nice relationship of $f_{0}(q)$ with the little $q$-Jacobi polynomials [12; p. 27].

It should be emphasized that there is more in common than just parity considerations between the refinements of Gordon's Theorem (Sections 2-5) and parity indices (Sections 6-12). Indeed comparison of Theorem 4 at $k=2$ and Theorem 18 reveals that we are considering instances of

$$
\sum_{n=0}^{\infty} \frac{q^{n^{2}} x^{n} f_{n}(y ; q)}{\left(q^{2} ; q^{2}\right)_{n}}
$$

where we want $f_{n}(0 ; q)=1$ and $f_{n}(1 ; q)=(-q ; q)_{n}$.

For such polynomials we see that $y=0$ leads to Euler's series

$$
\sum_{n=0}^{\infty} \frac{q^{n^{2}} x^{n}}{\left(q^{2} ; q^{2}\right)_{n}}
$$

and $y=1$ leads to the Rogers-Ramanujan series

$$
\sum_{n=0}^{\infty} \frac{q^{n^{2}} x^{n}}{(q ; q)_{n}}
$$

This entire project began with the observation that there are two obvious choices for $f$, namely $H_{n}$, the Rogers-Szegö polynomial (defined in Section 5) and $(-y q ; q)_{n}$. The first choice leads naturally to Theorem 4 and subsequently to everything in Sections 2-5; the latter choice leads to the material in Sections 6-12.

Finally we note that K. Kursungoz has made an extensive combinatorial study of many of the the theorems in this paper and has obtained corresponding bijective or sieve-theoretic proofs. We will catalog his achievements in Section 13.

\section{BACKGROUND}

In subsequent sections, we require a number of results and techniques from the literature. We shall collect all those elements in this section.

Our proof of Theorems 1 and 2 will require the following. As in [1], we define

$$
\begin{gathered}
C_{k, i}(x ; q)=\sum_{n=0}^{\infty}(-1)^{n} x^{k n} q^{\frac{1}{2}(2 k+1) n(n+1)-i n} \frac{\left(1-x^{i} q^{(2 n+1) i}\right)(x q ; q)_{n}}{(q ; q)_{n}}, \\
Q_{k, i}(x ; q)=\frac{C_{k, i}(x ; q)}{(x q ; q)_{\infty}}
\end{gathered}
$$


It was shown in [1] and [3], that

$$
\begin{gathered}
Q_{k, 0}(x ; q)=0, \\
Q_{k, k+1}(x ; q)=Q_{k, k}(x ; q), \\
Q_{k,-i}(x ; q)=-(x q)^{-i} Q_{k, i}(x ; q), \\
Q_{k, i}(x ; q)-Q_{k, i-1}(x ; q)=x^{i-1} q^{i-1} Q_{k, k-i+1}(x q ; q) .
\end{gathered}
$$

The functions $Q_{k, i}(x ; q)$ play a vital role in the proof of the Rogers-RamanujanGordon identities given in the introduction. Namely, if we let $b_{k, a}(m, n)$ denote the number of partitions enumerated by $B_{k, a}(n)$ that have exactly $m$ parts, then [1]

$$
Q_{k, a}(x ; q)=\sum_{m, n \geqq 0} b_{k, a}(m, n) x^{m} q^{n} .
$$

In addition (cf. [1] or [3])

$$
Q_{k, a}(1 ; q)=\frac{\left(q^{a} ; q^{2 k+1}\right)_{\infty}\left(q^{2 k+1-a} ; q^{2 k+1}\right)_{\infty}\left(q^{2 k+1} ; q^{2 k+1}\right)_{\infty}}{(q ; q)_{\infty}} .
$$

In [6], it was shown that

$$
Q_{k, i}(x ; q)=\sum_{n_{1}, \ldots, n_{k} \geqq 0} \frac{q^{N_{1}^{2}+N_{2}^{2}+\cdots+N_{k-1}^{2}+N_{i}+N_{i+1}+\cdots+N_{k-1}} x^{N_{1}+N_{2}+\cdots+N_{k-1}}}{(q ; q)_{n_{1}}(q ; q)_{n_{2}} \cdots(q ; q)_{n_{k-1}}} .
$$

We also require the $q$-binomial coefficients

$$
\left[\begin{array}{l}
N \\
M
\end{array}\right]_{k}=\left\{\begin{array}{ll}
0 & \text { if } M<0 \text { or } M>N \\
\frac{\left(q^{k} ; q^{k}\right)_{N}}{\left(q^{k} ; q^{k}\right)_{M}\left(q^{k} ; q^{k}\right)_{N-M}} & \text { otherwise }
\end{array} .\right.
$$

These satisfy two basic recurrences [7; p. 35, eqs. (3.3.4) and (3.3.3)]

$$
\begin{aligned}
& {\left[\begin{array}{l}
N \\
M
\end{array}\right]_{k}=\left[\begin{array}{l}
N-1 \\
M-1
\end{array}\right]_{k}+q^{k M[}\left[\begin{array}{c}
N-1 \\
M
\end{array}\right]_{k}} \\
& {\left[\begin{array}{l}
N \\
M
\end{array}\right]_{k}=\left[\begin{array}{c}
N-1 \\
M
\end{array}\right]_{k}+q^{k(N-M)}\left[\begin{array}{l}
N-1 \\
M-1
\end{array}\right]_{k}}
\end{aligned}
$$

We conclude this section with some remarks about standard methods used for partition generating functions.

Often we will refer to $f(x ; q)$ as the generating function for all partitions subject to certain constraints $\mathcal{C}$. By this we mean that if $P_{\mathcal{C}}(m, n)$ is the number of partitions of $n$ into $m$ summands subject to the constraint $\mathcal{C}$, then

$$
f(x ; q)=\sum_{m, n \geqq 0} P_{\mathcal{C}}(m, n) x^{m} q^{n} .
$$

Next we shall often use the Shift Rule. Namely

$$
f\left(x q^{j} ; q\right)
$$

is the generating function for partitions subject to $\mathcal{C}$ wherein each part has had $j$ added to it.

Additionally we often shall consider that the partitions subject to constraint $\mathcal{C}$ must also have all their summands $\leqq N$. The generating function will now be denoted by $f(N, x ; q)$. 
Often we will obtain recurrences for $f(N, x ; q)$ utilizing the Largest Part Decomposition Principle as follows:

$$
\begin{aligned}
f(N, x ; q)= & f(N-1, x ; q) \\
& + \text { generating function for those partitions subject to } \mathcal{C} \text { and } \\
& \text { having } N \text { as a summand. }
\end{aligned}
$$

Next let us suppose that adding a given $j$ to each part of every partition constrained by $\mathcal{C}$ produces exactly all those partitions constrained by $\mathcal{C}$ whose parts are $>j$. Under these circumstances we may have a Smallest Parts Decomposition Principle as follows:

$$
\begin{aligned}
f(x ; q)= & f\left(x q^{j} ; q\right) \\
& + \text { generating function for those partitions subject to } \mathcal{C} \text { and } \\
& \text { having at least one summand } \leqq j .
\end{aligned}
$$

Finally suppose that we have two sets of functions $f_{i}(x, q), 1 \leqq i \leqq r$ and $g_{i}(x, q)$, $1 \leqq i \leqq r$ which are analytic in $x$ and $q$ for $|q|<1$ and $|x|<|q|^{-1}$. Furthermore suppose that for each $i, f_{i}(0, q)=g_{i}(0, q)$,

$$
f_{i}(x, q)=\sum_{j=1}^{r} h_{i, j}(x, q) f_{j}\left(x q^{e(i, j)}, q\right)
$$

and

$$
g_{i}(x, q)=\sum_{j=1}^{r} h_{i, j}(x, q) g_{j}\left(x q^{e(i, j)}, q\right)
$$

where the $e(i, j)$ are all positive integers and the $h_{i, j}(x, q)$ are polynomials in $x$ and $q$. Then it follows by a double induction on the double power series coefficients that

$$
f_{i}(x, q)=g_{i}(x, q), \quad 1 \leqq i \leqq r .
$$

We shall refer to this process of identifying two sets of functions as the Defining q-Difference Equations Principle.

When we invoke this principle, it may be the case that linear combinations of the given functional equations are necessary to fulfill precisely (2.13) and (2.14).

\section{THEOREMS 1 AND 2}

Suppose we let $w_{k, a}(m, n)$ (resp. $\left.\bar{w}_{k, a}(m, n)\right)$ denote the number of partitions of the type enumerated by $W_{k, a}(n)$ (resp. $\left.\bar{W}_{k, a}(n)\right)$ that have exactly $m$ parts. The related generating functions are

$$
\mathcal{W}_{k, a}(x ; q):=\sum_{m, n \geqq 0} w_{k, a}(m, n) x^{m} q^{n},
$$

and

$$
\overline{\mathcal{W}}_{k, a}(x ; q):=\sum_{m, n \geqq 0} \bar{w}_{k, a}(m, n) x^{m} q^{n} .
$$

Our first object will be to show that

$$
\mathcal{W}_{k, a}(x ; q)=\left(-x q ; q^{2}\right)_{\infty} Q_{\frac{k}{2}, \frac{a}{2}}\left(x^{2} ; q^{2}\right)
$$

provided $k \equiv a(\bmod 2)$, and when $k$ is odd and $a$ even,

$$
\overline{\mathcal{W}}_{k, a}(x ; q)=\left(-x q ; q^{2}\right)_{\infty} Q_{\frac{k}{2}, \frac{a}{2}}\left(x^{2} ; q^{2}\right) \text {. }
$$


We begin with $k$ even (and thus only consider (3.3).

Case 1 . The even case. Here we replace $k$ by $2 k$ and $a$ by $2 a$, and consequently we need (3.2) to show that

$$
\mathcal{W}_{2 k, 2 a}(x ; q):=\left(-x q ; q^{2}\right)_{\infty} Q_{k, a}\left(x^{2} ; q^{2}\right) .
$$

We start off by adding together instances of (2.6) for $1 \leqq i \leqq a$ and noting $Q_{k, 0}\left(x^{2} ; q^{2}\right)=0$ by $(2.3)$, thus by $(2.6)$

$$
\begin{aligned}
Q_{k, a}\left(x^{2} ; q^{2}\right) & =\sum_{i=1}^{a}\left(x^{2} q^{2}\right)^{i-1} Q_{k, k-i+1}\left(x^{2} q^{2} ; q^{2}\right) \\
& =\sum_{i=1}^{a}\left(x^{2} q^{2}\right)^{i-1} \sum_{h=1}^{k-i+1}\left(x^{2} q^{4}\right)^{h-1} Q_{k, k-h+1}\left(x^{2} q^{4} ; q^{2}\right) .
\end{aligned}
$$

Now defining

$$
V_{2 k, 2 a}(x ; q)=\left(-x q ; q^{2}\right)_{\infty} Q_{k, a}\left(x^{2} ; q^{2}\right),
$$

we see that (3.6) implies

$$
\begin{aligned}
& V_{2 k, 2 a}(x ; q)= \\
& (1+x q) \sum_{i=1}^{a} x^{2(i-1)} q^{\overbrace{1+1+\ldots+1}^{2 i-2}} \sum_{h=1}^{k-i+1} x^{2(h-1)} q^{\overbrace{2+2+\ldots+2}^{2 h-2}} V_{2 k, 2 k-2 h+2}\left(x q^{2} ; q\right) .
\end{aligned}
$$

However, we may establish combinatorially that

$$
\begin{aligned}
& \mathcal{W}_{2 k, 2 a}(x ; q)= \\
& \sum_{i=1}^{a}(x^{2(i-1)} q^{\overbrace{1+1+\ldots+1}^{2 i-2}}+x^{2 i-1} q^{\overbrace{}^{\text {times }}} \overbrace{}^{2 i-1+\ldots+1}) \sum_{h=1}^{k-i+1} x^{2(h-1)} q^{2+2+\ldots+2} \mathcal{W}_{2 k, 2 a}\left(x q^{2} ; q\right) .
\end{aligned}
$$

To see this we note that if in the partitions enumerated by $w_{k, a}(m, n) 1$ appears $2 i-2$ times and 2 appears $2 h-2$ times, then 3 can appear at most $2 k-(2 h-2)-1=$ $(2 k-2 h+2)-1$ times. Thus by the Shift Rule, this particular set of partitions is generated by

$$
x^{2 i-2} q^{2 i-2} x^{2 h-2} q^{4 h-4} \mathcal{W}_{2 k, 2 k-2 h+2}\left(x q^{2} ; q\right) .
$$

In precisely the same way, those partitions enumerated by $w_{k, a}(m, n)$ in which 1 appears $2 i-1$ times and 2 appears $2 h-2$ times have as their generating function

$$
x^{2 i-1} q^{2 i-1} x^{2 h-2} q^{4 h-4} \mathcal{W}_{2 k, 2 k-2 h+2}\left(x q^{2} ; q\right) \text {. }
$$

Now summing over $1 \leqq i \leqq a$ and $1 \leqq h \leqq k-i+1$, we obtain (3.9). Thus by Defining $q$-Difference Equations Principle

$$
V_{2 k, 2 a}(x ; q)=\mathcal{W}_{2 k, 2 a}(x ; q)
$$

which establishes (3.3) in the even case.

Case 2. The odd case. This case is somewhat less intricate because we can combine the proof of (3.3) and (3.4) in this case. Namely replacing $k$ by $2 k+1$ and $a$ by $2 a+1$ in (3.3) and by $2 a$ in (3.4), we see that we need to prove

$$
\mathcal{W}_{2 k+1,2 a+1}(x ; q)=\left(-x q ; q^{2}\right)_{\infty} Q_{k+\frac{1}{2}, a+\frac{1}{2}}\left(x^{2} ; q^{2}\right)
$$


and

$$
\overline{\mathcal{W}}_{2 k+1,2 a}(x ; q)=\left(-x q^{2} ; q^{2}\right)_{\infty} Q_{k+\frac{1}{2}, a}\left(x^{2} ; q^{2}\right)
$$

Following the example of the previous case, we define

$$
V_{2 k+1,2 a+1}(x ; q)=\left(-x q ; q^{2}\right)_{\infty} Q_{k+\frac{1}{2}, a+\frac{1}{2}}\left(x^{2} ; q^{2}\right)
$$

and

$$
\bar{V}_{2 k+1,2 a}(x ; q)=\left(-x q^{2} ; q^{2}\right)_{\infty} Q_{k+\frac{1}{2}, a}\left(x^{2} ; q^{2}\right) .
$$

We shall next derive a defining set of $q$-difference equations and initial conditions for the $V$ 's and $\bar{V}$ 's. First by $(2.3)$

$$
\bar{V}_{2 k+1,0}(x ; q)=0
$$

and otherwise

$$
1=V_{2 k+1,2 a+1}(0 ; q)=V_{2 k+1,2 a+1}(x ; 0)=\bar{V}_{2 k+1,2 a}(0 ; q)=\bar{V}_{2 k+1,2 a}(x ; 0) .
$$

As for the $q$-difference equations

$$
V_{2 k+1}(x ; q)=\left(-x q ; q^{2}\right) Q_{k+\frac{1}{2}, \frac{1}{2}}\left(x^{2} ; q^{2}\right),
$$

and by (2.5) and (2.6),

$$
Q_{k+\frac{1}{2}, \frac{1}{2}}\left(x^{2} ; q^{2}\right)\left(1+\left(x^{2} q^{2}\right)^{-\frac{1}{2}}\right)=\left(x^{2} q^{2}\right)^{-\frac{1}{2}} Q_{k+\frac{1}{2}, k+1}\left(x^{2} q^{2} ; q^{2}\right) ;
$$

so

$$
V_{2 k+1,1}(x ; q)=\bar{V}_{2 k+1,2 k+2}(x q ; q) .
$$

For $0<a \leqq k$

$$
\begin{aligned}
V_{2 k+1,2 a+1}(x ; q) & -V_{2 k+1,2 a-1}(x ; q) \\
& =\left(-x q ; q^{2}\right)_{\infty}\left(Q_{k+\frac{1}{2}, a+\frac{1}{2}}\left(x^{2} ; q^{2}\right)-Q_{k+\frac{1}{2}, a-\frac{1}{2}}\left(x^{2} ; q^{2}\right)\right) \\
& =\left(-x q ; q^{2}\right)_{\infty} Q_{k+\frac{1}{2}, k-a+1}\left(x^{2} q^{2} ; q^{2}\right)\left(x^{2} q^{2}\right)^{a-\frac{1}{2}} \\
& =(x q)^{2 a-1}(1+x q) \bar{V}_{2 k+1,2 k-2 a+2}(x q ; q),
\end{aligned}
$$

and

$$
\begin{aligned}
\bar{V}_{2 k+1,2 a+2}(x ; q) & -\bar{V}_{2 k+1,2 a}(x ; q) \\
= & \left(-x q^{2} ; q^{2}\right)_{\infty}\left(Q_{k+\frac{1}{2}, a+1}\left(x^{2} ; q^{2}\right)-Q_{k+\frac{1}{2}, a}\left(x^{2} ; q^{2}\right)\right) \\
= & \left(-x q^{2} ; q^{2}\right)_{\infty}\left(x^{2} q^{2}\right)^{a} Q_{k+\frac{1}{2}, k-a+\frac{1}{2}}\left(x^{2} q^{2} ; q^{2}\right) \\
= & (x q)^{2 a} V_{2 k+1,2 k-2 a+1}(x q ; q) .
\end{aligned}
$$

The initial conditions (3.15) and (3.16) plus the $q$-difference equations (3.17) and (3.18) will allow us to identify the $V$ 's with the $W$ 's provided we prove that the $W$ 's satisfy the same conditions.

\section{Clearly}

$$
\bar{W}_{2 k+1,0}(x ; q)=0
$$

because there are no partitions with $\leqq-1$ appearances of 1 .

Also

$(3.21) 1=W_{2 k+1,2 a+1}(0 ; q)=W_{2 k+1,2 a+1}(x ; 0)=\bar{W}_{2 k+1,2 a}(0 ; q)=\bar{W}_{2 k+1,2 a}(x ; 0)$ because in each case the only partition allowed is the empty partition of 0 . 
Now

$$
w_{2 k+1,2 a+1}(m, n)-w_{2 k+1,2 a-1}(m, n)
$$

counts those partitions enumerated by $w_{2 k+1,2 a+1}(m, n)$ with the added condition that 1 appears either $2 a$ times or $2 a-1$ times. We transform these partitions by deleting all the 1 's and subtracting 1 from each of the remaining summands. If there were initially $2 a$ ones, then there were initially at most $(2 k-2 a+1)-1$ twos (remember two cannot appear an odd number of times), and subtracting 1 from each part changes the parity of each part. Hence this first class of partitions is enumerated by

$$
\bar{w}_{2 k+1,2 k-2 a+2}(m-2 a, n-m) .
$$

If there were initially $2 a-1$ ones, then there were at most $(2 k-2 a+2)-1$ twos. Hence this second class of partitions is enumerated by

$$
\bar{w}_{2 k+1,2 k-2 a+2}(m-2 a+1, n-m) .
$$

Putting this all together, we see that

$$
\begin{aligned}
& w_{2 k+1,2 a+1}(m, n)-w_{2 k+1,2 a-1}(m, n) \\
& \quad=\bar{w}_{2 k+1,2 k-2 a+2}(m-2 a, n-m)+\bar{w}_{2 k+1,2 k-2 a+2}(m-2 a+1, n-m),
\end{aligned}
$$

and (3.22) may be translated to the related generating functions:

$$
\begin{array}{r}
W_{2 k+1,2 a+1}(x ; q)-W_{2 k+1,2 a-1}(x ; q) \\
=(x q)^{2 a-1}(1+x q) W_{2 k+1,2 k-2 a+2}(x q ; q),
\end{array}
$$

which is (3.18) for the $W$ 's. Finally

$$
\bar{w}_{2 k+1,2 a+2}(m, n)-\bar{w}_{2 k+1,2 a}(m, n)
$$

counts those partitions enumerated by $\bar{w}_{2 k+1,2 a+2}(m, n)$ with the added condition that 1 appear $2 a$ times (keep in mind that 1 , being odd, cannot appear $2 a+1$ times). We transform these partitions by deleting the 1's and subtracting 1 from each of the remaining summands. Since 1 appears $2 a$ times, then there were initially at most $(2 k+1-2 a)-1$ twos. As before, subtracting 1 from each part changes the parity. Hence the transformed partitions are enumerated by

$$
w_{2 k+1,2 k-2 a+1}(m-2 a, n-m) .
$$

Therefore

$$
\bar{w}_{2 k+1,2 a+2}(m, n)-\bar{w}_{2 k+1,2 a}(m, n)=w_{2 k+1,2 k-2 a+1}(m-2 a, n-m),
$$

and (3.24) may be translated to the related generating functions

$$
\bar{W}_{2 k+1,2 a+1}(x ; q)-\bar{W}_{2 k+1,2 a-1}(x ; q)=(x q)^{2 a} W_{2 k+1,2 k-2 a+1}(x q ; q)
$$

which is (3.19) for the $W$ 's.

Hence by the Defining $q$-Difference Equations Principle, the $W$ 's and the $V$ 's are identical. I.e. (3.3) and (3.4) are valid. 
The proofs of Theorem 1 and 2 are now quite straight forward.

$$
\begin{aligned}
\sum_{n=0}^{\infty} \mathcal{W}_{k, a}(n) q^{n} & =\sum_{n, m \geqq 0} w_{k, a}(m, n) q^{n} \\
& =W_{k, a}(1, q)=\left(-q ; q^{2}\right)_{\infty} Q_{\frac{k}{2}, \frac{a}{2}}\left(1 ; q^{2}\right) \\
& =\frac{\left(-q ; q^{2}\right)_{\infty}\left(q^{a} ; q^{2 k+2}\right)_{\infty}\left(q^{2 k+2-a} ; q^{2 k+2}\right)_{\infty}\left(q^{2 k+2} ; q^{2 k+2}\right)_{\infty}}{\left(q^{2} ; q^{2}\right)_{\infty}} \\
& =\frac{\left(q^{a} ; q^{2 k+2}\right)_{\infty}\left(q^{2 k+2-a} ; a^{2 k+2}\right)_{\infty}\left(q^{2 k+2} ; q^{2 k+2}\right)_{\infty}}{\left(q ; q^{2}\right)_{\infty}\left(q^{4} ; q^{4}\right)_{\infty}} \\
& =\sum_{n=0}^{\infty} G_{k, a}(n) q^{n} .
\end{aligned}
$$

The last line follows from the antepenultimate line if $k$ an $a$ are both even and from the penultimate line if $k$ and $a$ are both odd. Comparing coefficients in the extremes of (3.26), we see that Theorem 1 is proved.

Finally for $k$ odd and $a$ even,

$$
\begin{aligned}
\sum_{n=0}^{\infty} \overline{\mathcal{W}}_{k, a}(n) q^{n} & =\sum_{n, m \geqq 0} \bar{w}_{k, a}(n) q^{n} \\
& =\bar{W}_{k, a}(1, q)=\left(-q^{2} ; q^{2}\right)_{\infty} Q_{\frac{k}{2}, \frac{a}{2}}\left(1 ; q^{2}\right) \\
& =\frac{\left(q^{a} ; q^{2 k+2}\right)_{\infty}\left(q^{2 k+2-a} ; q^{2 k+2}\right)_{\infty}\left(q^{2 k+2} ; q^{2 k+2}\right)_{\infty}}{\left(-q ; q^{2}\right)_{\infty}(q ; q)_{\infty}}
\end{aligned}
$$

by $(2.8)$ as asserted in Theorem 2 .

While there is no really clean classical partition-theoretic interpretation of (3.27), it can be interpreted via overpartitions (cf. [11], [18]). Also the equivalent assertion

$$
\left(1+2 \sum_{n=1}^{\infty}(-1)^{n} q^{2 n^{2}}\right) \sum_{n=0}^{\infty} \overline{\mathcal{W}}_{k, a}(n) q^{n}=\sum_{n=-\infty}^{\infty}(-1)^{n} q^{(k+1) n(n-1)+a n}
$$

yields a very nice recurrence for $\overline{\mathcal{W}}_{k, a}(n)$.

\section{THEOREM 3}

In light of (3.2) and Theorem 1 we shall first prove that

$$
\begin{aligned}
\left(-q ; q^{2}\right)_{\infty} Q_{\frac{k}{2}, \frac{a}{2}} & \left(1 ; q^{2}\right) \\
& =\sum_{n_{1}, \ldots, n_{k-1} \geqq 0} \frac{q^{N_{1}^{2}+N_{2}^{2}+\cdots+N_{k-1}^{2}+2 N_{a}+2 N_{a+2}+\cdots+2 N_{k-2}}}{\left(q^{2} ; q^{2}\right)_{n_{1}}\left(q^{2} ; q^{2}\right)_{n_{2}} \cdots\left(q^{2} ; q^{2}\right)_{n_{k-1}}},
\end{aligned}
$$

where, as noted earlier, $N_{i}=n_{i}+n_{i+1}+\cdots+n_{k-1}$.

Case 1. The even case. As before we replace $k$ by $2 k$ and $a$ by $2 a$. We shall prove that

$$
\begin{aligned}
& \left(-x q ; q^{2}\right)_{\infty} Q_{k, a}\left(x^{2} ; q^{2}\right) \\
& \quad=\sum_{n_{1}, \ldots, n_{2 k-1}=-\infty}^{\infty} \frac{q^{N_{1}^{2}+N_{2}^{2}+\cdots+N_{k-1}^{2}+2 N_{2 a}+2 N_{2 a+2}+\cdots+2 N_{2 k-2} x^{N_{1}+N_{2}+\cdots+N_{2 k-1}}}}{\left(q^{2} ; q^{2}\right)_{n_{1}}\left(q^{2} ; q^{2}\right)_{n_{2}} \cdots\left(q^{2} ; q^{2}\right)_{n_{2 k-1}}}
\end{aligned}
$$


Note that we sum from $-\infty$ to $\infty$ for convenience; indeed the extended definition $(q ; q)_{n}=\prod_{j=1}^{\infty}\left(1-q^{j}\right) /\left(1-q^{n+j}\right)$, means that $1 /(q ; q)_{n}=0$ if $n<0$. First we replace each $n_{2 i}$ by $n_{2 i}-n_{2 i+1}$ for $1 \leqq i \leqq k-1$. With this change of indices

$$
N_{2 i-1}=n_{2 i-1}+n_{2 i}+n_{2 i+2}+\cdots+n_{2 k-2}
$$

and

$$
N_{2 i}=n_{2 i}+n_{2 i+2}+\cdots+n_{2 k-2} .
$$

Thus we may rewrite the left-hand side of (4.2) as

$$
\begin{aligned}
\sum_{n_{2}, n_{4}, \ldots, n_{2 k-2}=-\infty}^{\infty} & \frac{q^{2 N_{2}^{2}+2 N_{4}^{2}+\cdots+2 N_{2 k-2}^{2}+2 N_{2 a}+2 N_{2 a+2}+\cdots+2 N_{2 k-2}} x^{2 N_{2}+2 N_{4}+\cdots+2 N_{2 k-2}}}{\left(q^{2} ; q^{2}\right)_{n_{2}}\left(q^{2} ; q^{2}\right)_{n_{4}} \cdots\left(q^{2} ; q^{2}\right)_{n_{2 k-2}}} \\
& \times \sum q^{n_{1}^{2}+2 n_{1} N_{2}+n_{3}^{2}+2 n_{3} N_{4}+\cdots+n_{2 k-3}^{2}+2 n_{2 k-3} N_{2 k-2}+n_{2 k-1}^{2}} x^{n_{1}+n_{3}+\cdots+n_{2 k-1}} \\
& \times n_{1}, n_{3}, \ldots, n_{2 k-1} \geqq 0 \\
& \times \frac{1}{\left(q^{2} ; q^{2}\right)_{n_{1}}}\left[\begin{array}{l}
n_{2} \\
n_{3}
\end{array}\right]_{2}\left[\begin{array}{l}
n_{4} \\
n_{5}
\end{array}\right]_{2} \ldots\left[\begin{array}{l}
n_{2 k-2} \\
n_{2 k-1}
\end{array}\right]_{2} \\
= & \sum \frac{q^{2 N_{2}^{2}+2 N_{4}^{2}+\cdots+2 N_{2 k-2}^{2}+2 N_{2 a}+\cdots+2 N_{2 k-2}} x^{2 N_{2}+\cdots+2 N_{2 k-2}}}{\left.n_{2}, n_{4}, \ldots, n_{2 k-2}=-q^{2} ; q^{2}\right)_{n_{2}}\left(q^{2} ; q^{2}\right)_{n_{4}} \cdots\left(q^{2} ; q^{2}\right)_{n_{2 k-2}}} \\
& \times\left(-x q^{1+2 N_{2}} ; q^{2}\right)_{\infty}\left(-x q^{1+2 N_{4}} ; q^{2}\right)_{n_{2}}\left(-x q^{1+2 N_{6}} ; q^{2}\right)_{n_{4}} \cdots\left(-x q ; q^{2}\right)_{n_{2 k-2}} \\
= & \left(-x q ; q^{2}\right)_{\infty} Q_{k, a}\left(x^{2} ; q^{2}\right),
\end{aligned}
$$

(by $[10 ;$ p. 36 , eq. (3.3.6)])

where we have combined the inner numerator factors into the single infinite product $\left(-x q ; q^{2}\right)_{\infty}$ and have invoked $(2.9)$.

Finally setting $x=1$ in (4.2) yields (4.1) in the even case.

Case 2. The odd case. Now we replace $k$ by $2 k+1$ and $a$ by $2 a+1$. We shall prove that

$$
\begin{aligned}
& \left(-x q ; q^{2}\right)_{\infty} Q_{k+\frac{1}{2}, a+\frac{1}{2}}\left(x^{2} ; q^{2}\right) \\
& \quad=\sum_{n_{1}, \ldots, n_{2 k} \geqq 0} \frac{q^{N_{1}^{2}+N_{2}^{2}+\cdots+N_{2 k}^{2}+2 N_{2 a+1}+2 N_{2 a+3}+\cdots+2 N_{2 k-1}} x^{N_{1}+N_{2}+\cdots+N_{2 k}}}{\left(q^{2} ; q^{2}\right)_{n_{1}}\left(q^{2} ; q^{2}\right)_{n_{2}} \cdots\left(q^{2} ; q^{2}\right)_{n_{2 k}}} .
\end{aligned}
$$

We now define

$$
R_{2 k+1,2 a+1}(x)=\left(-x q ; q^{2}\right)_{\infty} Q_{k+\frac{1}{2}, a+\frac{1}{2}}\left(x^{2} ; q^{2}\right),
$$

and

$$
R_{2 k+1,2 a+2}(x)=\left(-x q^{2} ; q^{2}\right)_{\infty} Q_{k+\frac{1}{2}, a+1}\left(x^{2} ; q^{2}\right) .
$$

Hence for $k+1 \geqq a>0$,

$$
\begin{aligned}
& R_{2 k+1,2 a+1}(x)-R_{2 k+1,2 a-1}(x) \\
& =\left(-x q ; q^{2}\right)_{\infty}\left(Q_{k+\frac{1}{2}, a+\frac{1}{2}}\left(x^{2} ; q^{2}\right)-Q_{k+\frac{1}{2}, a-\frac{1}{2}}\left(x^{2} ; q^{2}\right)\right) \\
& =\left(-x q ; q^{2}\right)_{\infty}\left(x^{2} q^{2}\right)^{a-\frac{1}{2}} Q_{k+\frac{1}{2}, k-a+1}\left(x^{2} q^{2} ; q^{2}\right) \\
& =(x q)^{2 a-1}(1+x q) R_{2 k+1,2 k-2 a+2}(x q)
\end{aligned}
$$


(note that for $a=k+1$, this implies $R_{2 k+1,2 k+3}(x)=R_{2 k+1,2 k+1}(x)$ ), for $k \geqq a \geqq 0$

$$
\begin{aligned}
R_{2 k+1,2 a+2}(x)-R_{2 k+1,2 a}(x) & =\left(-x q^{2} ; q^{2}\right)_{\infty}\left(Q_{k+\frac{1}{2}, a+1}\left(x^{2} ; q^{2}\right)-Q_{k+\frac{1}{2}, a}\left(x^{2} ; q^{2}\right)\right) \\
& =(x q)^{2 a}\left(-x q^{2} ; q^{2}\right)_{\infty} Q_{k+\frac{1}{2}, k-a+\frac{1}{2}}\left(x^{2} q^{2} ; q^{2}\right) \\
& =(x q)^{2 a} R_{2 k+1,2 k-2 a+1}(x q),
\end{aligned}
$$

and for $a=0$

$$
\begin{aligned}
R_{2 k+1,1}(x)\left(1+x^{-1} q^{-1}\right) & =R_{2 k+1,1}(x)-R_{2 k+1,-1}(x) \\
& =(x q)^{-1}(1+x q) R_{2 k+1,2 k+2}(x q),
\end{aligned}
$$

or

$$
R_{2 k+1,1}(x)=R_{2 k+1,2 k+2}(x q) .
$$

Now equations (4.6) - (4.8) are a defining set of $q$-difference equations once we note the obvious initial conditions for $x=0$ and $q=0$.

Furthermore, when $k=0$, the unique solutions are

$$
R_{1,1}(x)=R_{1,2}(x)=1, \quad R_{1,0}(x)=0 .
$$

We shall now prove that if for $0 \leqq a \leqq k$

$$
\mathcal{R}_{2 k+1,2 a+1}(x)=\sum_{n \geqq 0} \frac{\left(-x q ; q^{2}\right)_{n} q^{(2 k-1) n^{2}+2(k-a) n} x^{(2 k-1) n} R_{2 k-1,2 a+1}\left(x q^{2 n}\right)}{\left(q^{2} ; q^{2}\right)_{n}},
$$

and for $a=k+1$

$$
\begin{aligned}
\mathcal{R}_{2 k+1,2 k+3}(x) & =\sum_{n \geqq 0} \frac{\left(-x q ; q^{2}\right)_{n} q^{(2 k-1) n^{2}} x^{(2 n-1) n} R_{2 k-1,2 k-1}\left(x q^{2 n}\right)}{\left(q^{2} ; q^{2}\right)_{n}} \\
& =\mathcal{R}_{2 k+1,2 k+1}(x),
\end{aligned}
$$

and if for $0 \leqq a \leqq k$

$$
\mathcal{R}_{2 k+1,2 a}(x)=\sum_{n \geqq 0} \frac{\left(-x q^{2} ; q^{2}\right)_{n} q^{(2 k-1) n^{2}+(2 k-2 a+1) n} x^{(2 k-1) n} R_{2 k-1,2 a}\left(x q^{2 n}\right)}{\left(q^{2} ; q^{2}\right)_{n}},
$$

while

$$
\mathcal{R}_{2 k+1,2 k+2}(x)=\sum_{n \geqq 0} \frac{\left(-x ; q^{2}\right)_{n} q^{(2 k-1) n^{2}+n} x^{(2 k-1) n} R_{2 k-1,2 k}\left(x q^{2 n}\right)}{\left(q^{2} ; q^{2}\right)_{n}},
$$

then for $0 \leqq A \leqq 2 k+3, k>0$,

$$
\mathcal{R}_{2 k+1, A}(x)=R_{2 k+1, A}(x) .
$$


We now proceed to the base case, $k=1$, in order to establish (4.13) by mathematical induction on $k$. The asserted expansions for the $\mathcal{R}$ 's are:

$$
\begin{gathered}
\mathcal{R}_{3,1}(x)=\sum_{n \geqq 0} \frac{\left(-x q ; q^{2}\right)_{n} q^{n^{2}+2 n} x^{n}}{\left(q^{2} ; q^{2}\right)_{n}} \\
\mathcal{R}_{3,2}(x)=\sum_{n \geqq 0} \frac{\left(-x q^{2} ; q^{2}\right)_{n} q^{n^{2}+n} x^{n}}{\left(q^{2} ; q^{2}\right)_{n}} \\
\mathcal{R}_{3,5}(x)=\mathcal{R}_{3,3}(x)=\sum_{n \geqq 0} \frac{\left(-x q ; q^{2}\right)_{n} q^{n^{2}} x^{n}}{\left(q^{2} ; q^{2}\right)_{n}} \\
\mathcal{R}_{3,4}(x)=\sum_{n \geqq 0} \frac{\left(-x ; q^{2}\right)_{n} q^{n^{2}+n} x^{n}}{\left(q^{2} ; q^{2}\right)_{n}} .
\end{gathered}
$$

By inspection we see that,

$$
\begin{gathered}
\mathcal{R}_{3,5}(x)-\mathcal{R}_{3,3}(x)=0=x^{3}(1+x q) R_{3,0}(x q) \\
\mathcal{R}_{3,1}(x)=\mathcal{R}_{3,4}(x q) \\
\mathcal{R}_{3,2}(x)=\mathcal{R}_{3,3}(x q) .
\end{gathered}
$$

Also

$$
\begin{aligned}
\mathcal{R}_{3,3}(x)-\mathcal{R}_{3,1}(x) & =\sum_{n \geqq 1} \frac{\left(-x q ; q^{2}\right)_{n} q^{n^{2}} x^{n}}{\left(q^{2} ; q^{2}\right)_{n-1}} \\
& =x q(1+x q) \sum_{n \geqq 0} \frac{\left.\left(-(x q) q^{2} ; q^{2}\right)\right)_{n} q^{n^{2}+n}(x q)^{n}}{\left(q^{2} ; q^{2}\right)_{n}} \\
& =x q(1+x q) \mathcal{R}_{3,2}(x q),
\end{aligned}
$$

and

$$
\begin{aligned}
\mathcal{R}_{3,4}(x)-\mathcal{R}_{3,2}(x) & =\sum_{n \geqq 0} \frac{\left(-x q^{2} ; q^{2}\right)_{n-1} q^{n^{2}+n} x^{n}\left((1+x)-\left(1+x q^{2 n}\right)\right)}{\left(q^{2} ; q^{2}\right)_{n}} \\
& =x \sum_{n \geqq 1} \frac{\left(-x q^{2} ; q^{2}\right)_{n-1} q^{n^{2}+n} x^{n}}{\left(q^{2} ; q^{2}\right)_{n-1}} \\
& =x^{2} q^{2} \sum_{n \geqq 0} \frac{\left(-(x q) q ; q^{2}\right)_{n} q^{n^{2}+2 n}(x q)^{n}}{\left(q^{2} ; q^{2}\right)_{n}} \\
& =x^{2} q^{2} \mathcal{R}_{3,1}(x q) .
\end{aligned}
$$

Now (4.18) - (4.22) are just (4.6), (4.7) and (4.8) in the case $k=1$. Thus, noting the concurrent initial conditions, we see that by the Defining $q$-Difference Equation Principle, (4.13) is valid for $k=1$. 
We now assume that (4.13) is valid up to but not including a fixed $k$. Hence

$$
\mathcal{R}_{2 k+1,1}(x)=\sum_{n \geqq 0} \frac{\left(-x q ; q^{2}\right)_{n} q^{(2 k-1) n^{2}+2 k n} x^{(2 k-1) n} R_{2 k-1,1}\left(x q^{2 n}\right)}{\left(q^{2} ; q^{2}\right)_{n}}
$$

$$
\begin{aligned}
& =\sum_{n \geqq 0} \frac{\left(-x q ; q^{2}\right)_{n} q^{(2 k-1) n^{2}+n}(x q)^{(2 k-1) n} R_{2 k-1,2 k}\left(x q^{2 n+1}\right)}{\left(q^{2} ; q^{2}\right)_{n}} \\
& =\mathcal{R}_{2 k+1,2 k+2}(x q) .
\end{aligned}
$$

So (4.8) is valid with the $R$ 's replaced by $\mathcal{R}$ 's.

Next for $1 \leqq a<k$,

$$
\begin{aligned}
& \mathcal{R}_{2 k+1,2 a+1}(x)-\mathcal{R}_{2 k+1,2 a-1}(x) \\
= & \sum_{n \geqq 0} \frac{\left(-x q ; q^{2}\right)_{n} q^{(2 k-1) n^{2}+2(k-a) n} x^{(2 k-1) n}}{\left(q^{2} ; q^{2}\right)_{n}}\left(R_{2 k-1,2 a+1}\left(x q^{2 n}\right)-q^{2 n} R_{2 k-1,2 a-1}\left(x q^{2 n}\right)\right) \\
= & \sum_{n \geqq 0} \frac{\left(-x q ; q^{2}\right)_{n} q^{(2 k-1) n^{2}+2(k-a) n} x^{(2 k-1) n}}{\left(q^{2} ; q^{2}\right)_{n}} \\
& \times\left(R_{2 k-1,2 a-1}\left(x q^{2 n}\right)+\left(x q^{2 n+1}\right)^{2 a-1}\left(1+x q^{2 n+1}\right) R_{2 k-1,2 k-2 a}\left(x q^{2 n+1}\right)\right.
\end{aligned}
$$

(by $(4.6))$

$$
\begin{aligned}
& \left.-q^{2 n} R_{2 k-1,2 a-1}\left(x q^{2 n}\right)\right) \\
= & \sum_{n \geqq 1} \frac{\left(-x q ; q^{2}\right)_{n} q^{(2 k-1) n^{2}+2(k-a) n} x^{(2 k-1) n} R_{2 k-1,2 a-1}\left(x q^{2 n}\right)}{\left(q^{2} ; q^{2}\right)_{n-1}} \\
& +(x q)^{2 a-1}(1+x q) \sum_{n \geqq 0} \frac{\left(-(x q) q^{2} ; q^{2}\right)_{n} q^{(2 k-1) n^{2}+(2 a-1) n} x^{(2 k-1) n} R_{2 k-1,2 k-2 a}\left(x q^{2 n}\right)}{\left(q^{2} ; q^{2}\right)_{n}} \\
= & \sum_{n \geqq 1} \frac{\left(-x q ; q^{2}\right)_{n+1} q^{(2 k-1)(n+1)^{2}+2(k-a)(n+1)} x^{(2 k-1)(n+1)} R_{2 k-1,2 a-1}\left(x q^{2 n+2}\right)}{\left(q^{2} ; q^{2}\right)_{n}} \\
& +(x q)^{2 a-1}(1+x q) \sum_{n \geqq 0} \frac{\left(-x q^{3} ; q^{2}\right)_{n} q^{(2 k-1) n^{2}+(2 a-1) n}(x q)^{(2 k-1) n}}{\left(q^{2} ; q^{2}\right)_{n}}
\end{aligned}
$$

(by $(4.7)$ )

$$
\times\left(R_{2 k-1,2 k-2 a+2}\left(x q^{2 n+1}\right)-\left(x q^{2 n+2}\right)^{2(k-a)} R_{2 k-1,2 a-1}\left(x q^{2 n+2}\right)\right)
$$

$$
=(x q)^{2 a-1}(1+x q) \mathcal{R}_{2 k+1,2 k-2 a+2}(x q),
$$

where the sums involving $R_{2 k-1,2 a-1}\left(x q^{2 n+1}\right)$ cancel each other. Hence we have (4.6) proved with $\mathcal{R}$ 's replacing $R$ 's as long as $1 \leqq a \leqq k$. At $a=k+1$

$$
\begin{aligned}
\mathcal{R}_{2 k+1,2 k+3}(x)-\mathcal{R}_{2 k+1,2 k+1}(x) & =0 \\
& =(x q)^{2 k+1}(1+x q) \mathcal{R}_{2 k+1,0}(x q)
\end{aligned}
$$

(by (4.5) because $Q_{k+\frac{1}{2}, 0}\left(x^{2} ; q^{2}\right)=0$ ). Hence (4.6) is proved in all cases with $\mathcal{R}$ 's replacing $R$ 's. 
Next for $0 \leqq a<k$

$$
\begin{aligned}
& \mathcal{R}_{2 k+1,2 a+2}(x)-\mathcal{R}_{2 k+1,2 a}(x) \\
& =\sum_{n \geqq 0} \frac{\left(-x q^{2} ; q^{2}\right)_{n} q^{(2 k-1) n^{2}+(2 k-2 a-1) n} x^{(2 k-1) n}}{\left(q^{2} ; q^{2}\right)_{n}} \\
& \quad \times\left(R_{2 k-1,2 a+2}\left(x q^{2 n}\right)-q^{2 n} R_{2 k-1,2 a}\left(x q^{2 n}\right)\right) \\
& =\sum_{n \geqq 0} \frac{\left(-x q^{2} ; q^{2}\right)_{n} q^{(2 k-1) n^{2}+(2 k-2 a-1) n} x^{(2 k-1) n}}{\left(q^{2} ; q^{2}\right)_{n}} \\
& \quad \times\left(R_{2 k-1,2 a}\left(x q^{2 n}\right)+\left(x q^{2 n+1}\right)^{2 a} R_{2 k-1,2 k-2 a-1}\left(x q^{2 n+1}\right)-q^{2 n} R_{2 k-1,2 a}\left(x q^{2 n}\right)\right)
\end{aligned}
$$

(by $(4.7))$

$$
\begin{aligned}
= & \sum_{n \geqq 1} \frac{\left(-x q^{2} ; q^{2}\right)_{n} q^{(2 k-1) n^{2}+(2 k-2 a-1) n} x^{(2 k-1) n} R_{2 k-1,2 a}\left(x q^{2 n}\right)}{\left(q^{2} ; q^{2}\right)_{n-1}} \\
& +(x q)^{2 a} \sum_{n \geqq 0} \frac{\left(-x q^{2} ; q^{2}\right)_{n} q^{(2 k-1) n^{2}+(2 k-2 a-1) n+4 n a} x^{(2 k-1) n}}{\left(q^{2} ; q^{2}\right)_{n}} \\
& \times\left(R_{2 k-1,2 k-2 a+1}\left(x q^{2 n+1}\right)-\left(x q^{2 n+2}\right)^{2 k-2 a-1}\left(1+x q^{2 n+2}\right) R_{2 k-1,2 a}\left(x q^{2 n+2}\right)\right)
\end{aligned}
$$

(by $(4.6))$

$$
=(x q)^{2 a} \mathcal{R}_{2 k+1,2 k-2 a+1}(x q),
$$

where the two sums involving $R_{2 k-1,2 a}$ cancel each other out once $n$ has been replaced by $n+1$ in the first of the two. Hence we have (4.7) proved for $0 \leqq a<k$.

Finally for $a=k$

$$
\begin{aligned}
\mathcal{R}_{2 k+1,2 k+2}(x)-\mathcal{R}_{2 k+1,2 k}(x) \\
=\sum_{n \geqq 1} \frac{\left(-x q^{2} ; q^{2}\right)_{n-1} q^{(2 k-1) n^{2}+n} x^{(2 k-1) n} R_{2 k-1,2 k}\left(x q^{2 n}\right)}{\left(q^{2} ; q^{2}\right)_{n}}\left((1+x)-\left(1+x q^{2 n}\right)\right) \\
=x^{2 k} q^{2 k} \sum_{n \geqq 0} \frac{\left(-x q^{2} ; q^{2}\right)_{n} q^{(2 k-1) n^{2}+2 k n}(x q)^{(2 k-1) n} R_{2 k-1,2 k}\left(x q^{2 n+2}\right)}{\left(q^{2} ; q^{2}\right)_{n}} \\
=x^{2 k} q^{2 k} \sum_{n \geqq 0} \frac{\left(-(x q) q ; q^{2}\right)_{n} q^{(2 k-1) n^{2}+2 k n}(x q)^{(2 k-1) n} R_{2 k-1,1}\left(x q q^{2 n}\right)}{\left(q^{2} ; q^{2}\right)_{n}}
\end{aligned}
$$

(by $(4.8)$ )

$$
=(x q)^{2 k} \mathcal{R}_{2 k+1,1}(x q)
$$

which establishes (4.7) with the $R$ 's replaced by $\mathcal{R}$ 's. Hence by the Defining $q$ Difference Equations Principle (4.13) is true.

Hence (4.9) - (4.13) are valid if $\mathcal{R}$ is replaced by $R$.

I now claim that for $0 \leqq a \leqq k$

$$
R_{2 k+1,2 a+1}(x)=\sum_{n_{1}, \ldots, n_{2 k} \geqq 0} \frac{q^{N_{1}^{2}+\cdots+N_{2 k}^{2}+2 \sum_{j=a+1}^{k} N_{2 j-1}} x^{N_{1}+\cdots+N_{2 k}}}{\left(q^{2} ; q^{2}\right)_{N_{1}-N_{2}} \cdots\left(q^{2} ; q^{2}\right)_{N_{2 k-1}-N_{2 k}}\left(q^{2} ; q^{2}\right)_{N_{2 k}}}
$$


and

$$
=\sum_{n_{1}, \ldots, n_{2 k} \geqq 0} \frac{q^{N_{1}^{2}+\cdots+N_{2 k}^{2}+N_{2 a-1}+N_{2 a}+\cdots+N_{2 k}+n_{1}+n_{3}+n_{2 a-3}} x^{N_{1}+\cdots+N_{2 k}}}{\left(q^{2} ; q^{2}\right)_{N_{1}-N_{2}} \cdots\left(q^{2} ; q^{2}\right)_{N_{2 k-2}-N_{2 k-1}}\left(q^{2} ; q^{2}\right)_{N_{2 k-1}-N_{2 k}}\left(q^{2} ; q^{2}\right)_{N_{2 k}}},
$$

where $N_{i}=n_{i}+n_{i+1}+\cdots+n_{2 k}$ and empty sums are 0 .

If we denote the right hand side of $(4.26)$ by $\rho_{2 k+1,2 a+1}(x)$, we see that by summing the $n_{2 k}$ series using the $q$-binomial theorem [7; p. 36, eq. (3.3.6)] and letting $n=n_{2 k-1}$ we obtain

$$
\rho_{2 k+1,2 a+1}(x)=\sum_{n \geqq 0} \frac{\left(-x q ; q^{2}\right)_{n} q^{(2 k-1) n^{2}+2(k-a) n} x^{(2 k-1) n}}{\left(q^{2} ; q^{2}\right)_{n}} \rho_{2 k-1,2 a+1}\left(x q^{2 n}\right),
$$

an exact replica of (4.9) where we add the caveat that $\rho_{2 k+1,2 k+3}(x)=\rho_{2 k+1,2 k+1}(x)$ (i.e. $(4.10)$ ).

If we denote the right hand side of (4.7) by $\rho_{2 k+1,2 a}(x)$, we see that by summing the $n_{2 k}$ series using the $q$-binomial theorem [7; p. 36, eq. (3.36)] and letting $n=n_{2 k-1}$, we obtain

$$
\rho_{2 k+1,2 a}(x)=\sum_{n \geqq 0} \frac{\left(-x q^{2} ; q^{2}\right)_{n} q^{(2 k-1) n^{2}+(2 k-2 a+1) n} x^{(2 k-1) n} \rho_{2 k-1,2 a}\left(x q^{2 n}\right)}{\left(q^{2} ; q^{2}\right)_{n}}
$$

and

$$
\rho_{2 k+1,2 k+2}(x)=\sum_{n \geqq 0} \frac{\left(-x ; q^{2}\right)_{n} q^{(2 k-1) n^{2}+n} x^{(2 k-1) n} \rho_{2 k-1,2 k}\left(x q^{2 n}\right)}{\left(q^{2} ; q^{2}\right)_{n}} .
$$

Thus we have exactly the same functional equations for the $\rho$ 's as for the $R$ 's and exactly the same argument shows that

$$
\rho_{2 k+1, A}(x)=R_{2 k+1, A}(x)
$$

for $0 \leqq A \leqq 2 k+3$.

Now (4.4), (4.26) and (4.28) prove (4.2) in the odd case and consequently we have proved (4.1).

Finally to prove (1.9), the final assertion in Theorem 3 , we need only set $x=1$ in (4.5) and invoke (2.8).

\section{Evens Appearing Oddly in Gordon's Partitions}

Our object in this section is to prove the following result:

Theorem 4. If $k=2$ or 3 , then the coefficient of $q^{n} x^{m} y^{j}$ in

$$
\sum_{n_{1}, n_{2}, \ldots, n_{k-1} \geqq 0} \frac{q^{N_{1}^{2}+N_{2}^{2}+\cdots+N_{k-1}^{2}} x^{N_{1}+\cdots+N_{k-1}} H_{n_{1}} H_{n_{2}} \cdots H_{n_{k-1}}}{\left(q^{2} ; q^{2}\right)_{n_{1}}\left(q^{2} ; q^{2}\right)_{n_{2}} \cdots\left(q^{2} ; q^{2}\right)_{n_{k-1}}}
$$

is the number of partitions enumerated by $B_{k, k}(n)$ with exactly $m$ parts and exactly $j$ different even parts that appear an odd number of times. In (5.1),

$$
H_{n}=\sum_{j=0}^{n} y^{j} q^{j}\left[\begin{array}{l}
n \\
j
\end{array}\right]_{2}
$$


and as before

$$
N_{i}=n_{i}+n_{i+1}+\cdots+n_{k-1} .
$$

Noting that $H_{n}=1$ when $y=0$ and $H_{n}=(-q ; q)_{n}$ when $y=1$ [4; p. 49, Ex. $5]$, we see that the theorem is true for all $k$ when $y=1$ (it is the case $i=k$ of (2.9)) and when $y=0$ (it is the case $i=k$ of Theorem 3).

Remark. K. Kursungoz has found a combinatorial proof of (5.1) valid for all $k \geqq 2$. The proof here is purely analytic. The possibility of a fully analytic proof will be considered in Section 13.

To set up our proof we require a couple of lemmas. First we define

$$
\begin{aligned}
& S_{k}\left(a_{k-1}, a_{k-2}, \ldots, a_{1} ; x ; q\right) \\
& =\sum_{n_{1}, \ldots, n_{k-1} \geqq 0} \frac{q^{N_{1}^{2}+N_{2}^{2}+\cdots+N_{k-1}^{2}+2 a_{1} n_{1}+\cdots+2 a_{k-1} n_{k-1}} x^{N_{1}+\cdots+N_{k-1}} H_{n_{1}} H_{n_{2}} \cdots H_{n_{k-1}}}{\left(q^{2} ; q^{2}\right)_{n_{1}}\left(q^{2} ; q^{2}\right)_{n_{2}} \cdots\left(q^{2} ; q^{2}\right)_{n_{k-2}}}
\end{aligned}
$$

$$
=\sum_{n \geqq 0} \frac{q^{(k-1) n^{2}+2 a_{k-1} n} x^{(k-1) n} H_{n}}{\left(q^{2} ; q^{2}\right)_{n}} S_{k-1}\left(a_{k-2}, \ldots, a_{1} ; x q^{2 n} ; q\right) .
$$

Lemma 5. For $1 \leqq i \leqq k-1, k \geqq 2$,

$$
\begin{aligned}
& S_{k}\left(a_{k-1}, a_{k-2}, \ldots, a_{k-i}, \ldots, a_{1} ; x ; q\right)-S_{k}\left(a_{k-1}, a_{k-2}, \ldots, a_{k-i}+1, \ldots, a_{1} ; x ; q\right) \\
& =x^{k-i}(1+y q) q^{(k-i)+2 a_{k-i}} S_{k}\left(a_{k-1}-i+1, a_{k-2}-i+2, \ldots, a_{k-i+1}-1, a_{k-i}, \ldots, a_{1} ; x q^{2} ; q\right) \\
& -x^{2(k-i)} y q^{4\left(a_{k-i}+k-i\right)+1} S_{k}\left(a_{k-1}-2 i+2, a_{k-2}-2 i+4, \ldots, a_{k-i+1}-2, a_{k-i}, \ldots, a_{1} ; x q^{4} ; q\right) .
\end{aligned}
$$

Proof. We proceed by mathematical induction on $k$. First $k=2$. Consequently $i=1$. In the proof, we need the following recurrence for the $H$ 's [4; p. 49, Ex. 6]

$$
H_{n+1}=(1+y q) H_{n}-\left(1-q^{2 n}\right) y q H_{n-1} .
$$

Hence

$$
\begin{aligned}
& S_{2}\left(a_{1} ; x ; q\right)-S_{2}\left(a_{1}+1 ; x ; q\right) \\
& \quad=\sum_{n \geqq 0} \frac{q^{n^{2}+2 a_{1} n} x^{n} H_{n}\left(1-q^{2 n}\right)}{\left(q^{2} ; q^{2}\right)_{n}} \\
& =\sum_{n \geqq 0} \frac{q^{(n+1)^{2}+2 a_{1}(n+1)} x^{n+1} H_{n+1}}{\left(q^{2} ; q^{2}\right)_{n}} \\
& =\sum_{n \geqq 0} \frac{q^{(n+1)^{2}+2 a_{1}(n+1)} x^{n+1}\left((1+y q) H_{n}-\left(1-q^{2 n}\right) y q H_{n-1}\right)}{\left(q^{2} ; q^{2}\right)_{n}} \\
& =x q^{2 a_{1}+1}(1+y q) S_{2}\left(a_{1} ; x q^{2} ; q\right)-y q \sum_{n \geqq 0} \frac{q^{(n+2)^{2}+2 a_{1}(n+2)} x^{n+2} H_{n}}{\left(q^{2} ; q^{2}\right)_{n}} \\
& =x(1+y q) q^{1+2 a_{1}} S_{2}\left(a_{1} ; x q^{2} ; q\right)-x^{2} y q^{4\left(a_{1}+1\right)+1} S_{2}\left(a_{1} ; x q^{4} ; q\right) .
\end{aligned}
$$


Thus the case $k=2$ is established.

We now assume the theorem is true up to but not including a specific $k$.

When $i=1$

$$
\begin{aligned}
S_{k} & \left(a_{k-1}, a_{k-2}, \ldots, a_{1} ; x ; q\right)-S_{k}\left(a_{k-1}+1, a_{k-2}, \ldots, a_{1} ; x ; q\right) \\
= & \sum_{n \geqq 0} \frac{q^{(k-1) n^{2}+2 a_{k-1} n} x^{(k-1) n} H_{n}\left(1-q^{2 n}\right) S_{k-1}\left(a_{k-2}, \ldots, a_{1} ; x q^{2 n} ; q\right)}{\left(q^{2} ; q^{2}\right)_{n}} \\
= & \sum_{n \geqq 0} \frac{q^{(k-1)(n+1)^{2}+2 a_{k-1}(n+1)} x^{(k-1)(n+1)} S_{k-1}\left(a_{k-2}, \ldots, a_{1} ; x q^{2 n+2} ; q\right)}{\left(q^{2} ; q^{2}\right)_{n}} \\
& \times\left((1+y q) H_{n}-y q\left(1-q^{2 n}\right) H_{n-1}\right) \\
= & x^{k-1}(1+y q) q^{k-1+2 a_{k-1}} S_{k}\left(a_{k-1}, a_{k-2}, \ldots, a_{1} ; x q^{2} ; q\right) \\
& -y q \sum_{n \geqq 0} \frac{q^{(k-1)(n+2)^{2}+2 a_{k-1}(n+2)} x^{(k-1)(n+2)} H_{n}}{\left(q^{2} ; q^{2}\right)_{n}} S_{k-1}\left(a_{k-2}, \ldots, a_{1} ; x q^{2 n+4} ; q\right) \\
= & x^{k-1}(1+y q) q^{k-1+2 a_{k-1}} S_{k}\left(a_{k-1}, \ldots, a_{1} ; x q^{2} ; q\right) \\
& -x^{2(k-1)} y q^{1+4(k-1)+4 a_{k-1}} S_{k}\left(a_{k-1}, \ldots, a_{1} ; x q^{4} ; q\right)
\end{aligned}
$$

which is the desired result at $k$ with $i=1$. Now assume $k-1 \geqq i>1$,

$$
\begin{aligned}
& S_{k}\left(a_{k-1}, \ldots, a_{k-i}, \ldots, a_{1} ; x ; q\right)-S_{k}\left(a_{k-1}, \ldots, a_{k-i}+1, \ldots, a_{1} ; x ; q\right) \\
= & \sum_{n \geqq 0} \frac{q^{(k-1) n^{2}+2 a_{k-1} n} x^{(k-1) n} H_{n}}{\left(q^{2} ; q^{2}\right)_{n}}\left\{S_{k-1}\left(a_{k-2}, \ldots, a_{k-1-(i-1)}, \ldots, a_{1} ; x q^{2 n} ; q\right)\right. \\
& \left.-S_{k-1}\left(a_{k-2}, \ldots, a_{k-1-(i-1)}+1, \ldots, a_{1} ; x q^{2 n} ; q\right)\right\} \\
= & \sum_{n \geqq 0} \frac{q^{(k-1) n^{2}+2 a_{k-1} n} x^{(k-1) n} H_{n}}{\left(q^{2} ; q^{2}\right)_{n}} \\
\times & \left(\left(x q^{2 n}\right)^{k-i}(1+y q) q^{k-i+2 a_{k-i}} S_{k-1}\left(a_{k-2}-i+2, a_{k-3}-i+3, \ldots, a_{k-i}, \ldots, a_{1} ; x q^{2 n+2} ; q\right)\right. \\
& \left.-\left(x q^{2 n}\right)^{2(k-i)} y q^{4(k-i)+4 a_{k-i}+1} S_{k-1}\left(a_{k-2}-2 i+4, a_{k-3}-2 i+6, \ldots, a_{k-i}, \ldots, a_{1} ; x q^{2 n+4} ; q\right)\right)
\end{aligned}
$$

(by the induction hypothesis)

$$
\begin{aligned}
= & x^{k-i}(1+y q) q^{k-i+2 a_{k-i}} \\
& \times \sum_{n \geqq 0} \frac{q^{(k-1) n^{2}+2\left(a_{k-1}-i+1\right) n}\left(x q^{2}\right)^{(k-1) n} H_{n} S_{k-1}\left(a_{k-2}-i+2, \ldots ; x q^{2 n+2} ; q\right)}{\left(q^{2} ; q^{2}\right)_{n}} \\
& -x^{2(k-i)} y q^{4(k-i)+4 a_{k-i}+1} \\
& \times \sum_{n \geqq 0} q^{(k-1) n^{2}+2\left(a_{k-1}-2 i+2\right) n}\left(x q^{4}\right)^{(k-1) n} H_{n} S_{k-1}\left(a_{k-2}-2 i+4, \ldots ; x q^{2 n+4} ; q\right) \\
= & x^{k-i}(1+y q) q^{k-i+2 a_{k-i}} S_{k}\left(a_{k-1}-i+1, a_{k-2}-i+2, \ldots, a_{k-i}, \ldots, a_{1} ; x q^{2} ; q\right) \\
& -x^{2(k-i)} y q^{4(k-i)+4 a_{k-i}+1} S_{k}\left(a_{k-1}-2 i+2, a_{k-2}-2 i+4, \ldots, a_{k-i}, \ldots, a_{1} ; x q^{4} ; q\right)
\end{aligned}
$$

and so we have proved every instance at $k$ thus proving the lemma.

Next, 


\section{Lemma 6.}

(5.6) $S_{k}\left(a_{k-1}+k-1, a_{k-2}+k-2, \ldots, a_{1}+1 ; x ; q\right)=S_{k}\left(a_{k-1}, a_{k-2}, \ldots, a_{1} ; x q^{2} ; q\right)$.

Proof. This follows immediately from inspection of (5.2).

Finally,

Proof of Theorem 4. We begin with $k=2$. Let us define $G_{k}(x, y, q)$ to be the generating function for the partitions named in the statement of the theorem. It is easy to see that

$$
G_{2}(x, y, q)=\left(1+x q+x y q^{2}\right) G_{2}\left(x q^{2}, y, q\right)-x^{2} y q^{2+3} G_{2}\left(x q^{4}, y, q\right) .
$$

The first term on the right exhibits the allowable partitions accordingly as there are no parts smaller than 3 (the " 1 "), 1 is a part (the " $x q ")$, or 2 is a part (the " $x y q^{2} "$ ). However partitions with smallest parts $2+3$ have been inadmissably allowed. Thus the second term (with " $x^{2} y q^{2+3 ")}$ ) removes these partitions.

On the other hand, by Lemmas 5 and 6 ,

$$
\begin{aligned}
S_{2}(0 ; x ; q) & =S_{2}(1 ; x ; q)+x(1+y q) q S_{2}\left(0 ; x q^{2} ; q\right)-x^{2} y q^{5} S_{2}\left(0 ; x q^{4} ; q\right) \\
& =\left(1+x q+x y q^{2}\right) S_{2}\left(0 ; x q^{2} ; q^{2}\right)-x^{2} y q^{5} S_{2}\left(0 ; x q^{4} ; q\right) .
\end{aligned}
$$

Comparing (5.8) and (5.7), we see by the Defining $q$-Difference Equation Principle that

$$
G_{2}(x, y, q)=S_{2}(0 ; x ; q)
$$

This proves the case $k=2$.

We now consider $k=3$. We note that by Lemma 5

$$
S_{3}(0,0 ; x ; q)-S_{3}(1,0 ; x ; q)=x^{2} q^{2}(1+y q) S_{3}\left(0,0 ; x q^{2} ; q\right)-x^{4} y q^{9} S_{3}\left(0,0 ; x q^{4} ; q\right)
$$

$$
S_{3}(1,0 ; x ; q)-S_{3}(2,0 ; x ; q)=x^{2} q^{4}(1+y q) S_{3}\left(1,0 ; x q^{2} ; q\right)-x^{4} y q^{13} S_{3}\left(1,0 ; x q^{4} ; q\right)
$$

$$
S_{3}(2,0 ; x ; q)-S_{3}(2,1 ; x ; q)=x q(1+y q) S_{3}\left(1,0 ; x q^{2} ; q\right)-x^{2} y q^{5} S_{3}\left(0,0 ; x q^{4} ; q\right),
$$

and by Lemma 6 :

$$
S_{3}(2,1 ; x ; q)=S_{3}\left(0,0 ; x q^{2} ; q\right) .
$$

We may obtain $S_{3}(1,0 ; x ; q)$ in terms of instances of $S_{3}\left(0,0 ; x q^{2 i} ; q\right)$ from $(5.9)$. Then the same can be done for $S_{3}(2,0 ; x ; q)$ from $(5.10)$. Finally all of these results plus (5.12) can be substituted in (5.11) to obtain the following $q$-difference equation for $S_{3}(0,0 ; x ; q)$ :

$$
\begin{aligned}
& S_{3}(0,0 ; x ; q)=\left(1+q x(1+q y)\left(1+x q+x q^{3}\right)\right) S_{3}\left(0,0 ; x q^{2} ; q\right) \\
& +\left(x^{2} y q^{5}+x^{3} q^{7}(1+q y)^{2}+x^{4} q^{9}\left(q+y+2 q^{2} y+y q^{4}+y^{2} q^{3}\right)\right) S_{3}\left(0,0 ; x q^{4} ; q\right) \\
& \quad-x^{5} q^{18} y(1+y q)\left(1+x q^{3}+x q^{5}\right) S_{3}\left(0,0 ; x q^{6} ; q\right)+x^{8} y^{2} q^{38} S_{3}\left(0,0 ; x q^{8} ; q\right) .
\end{aligned}
$$

Now we need a $q$-difference equation for $G_{3}(x, y, q)$. This may be obtained as follows: $G_{3}(x, 1, q)$ is the generating function for the original Gordon partition $b_{3,3}(m, n)$. Following [3; p. 443, eqs. (5.6)-(5.9)], we see that the Smallest Parts Decomposition Principle may be invoked to yield

$$
G_{3}(x, 1, q)=(1+x q) G_{3}(x q, 1, q)-x^{2} q^{1+1} G_{3}\left(x q^{2}, 1, q\right)+x^{3} q^{2+2+1} G_{3}\left(x q^{3}, 1, q\right) .
$$


But in instances of $G_{3}\left(x q^{2 j+1}, y, q\right)$ we see that $y$ would be counting instances of different odd parts appearing an odd number of times. Hence we must do several applications of the shift rule in order to eliminate instances of $G_{3}\left(x q^{2 j+1}, 1, q\right)$. Thus with $G_{3}(x)=G_{3}(x, 1, q)$, we have that

$$
\begin{aligned}
& G_{3}(x q)=\left(1+x q^{2}\right) G_{3}\left(x q^{2}\right)-x^{2} q^{2+2} G_{3}\left(x q^{3}\right)+x^{3} q^{3+3+2} G_{3}\left(x q^{4}\right) \\
& G_{3}\left(x q^{2}\right)=\left(1+x q^{3}\right) G_{3}\left(x q^{3}\right)-x^{2} q^{3+3} G_{3}\left(x q^{4}\right)+x^{3} q^{4+4+3} G_{3}\left(x q^{4}\right) \\
& G_{3}\left(x q^{3}\right)=\left(1+x q^{4}\right) G_{3}\left(x q^{4}\right)-x^{2} q^{4+4} G_{3}\left(x q^{5}\right)+x^{4} q^{5+5+4} G_{3}\left(x q^{6}\right)
\end{aligned}
$$

We now have four linear equations which can be solved for $G_{3}(x), G_{3}(x q)$, $G_{3}\left(x q^{3}\right)$ and $G_{3}\left(x q^{5}\right)$ in terms of $G_{3}\left(x q^{2}\right), G_{3}\left(x q^{4}\right)$ and $G_{3}\left(x q^{6}\right)$. As a result

$$
\begin{array}{r}
G_{3}(x)=\left(1+x q+x^{2} q^{1+1}+x q^{2}+x^{2} q^{2+1}+x^{2} q^{2+2}\right) G_{3}\left(x q^{2}\right) \\
-\left(x^{3} q^{3+2+2}+x^{3} q^{3+3+2}+x^{4} q^{3+3+2+2}+x^{4} q^{3+3+2+1}+x^{4} q^{4+3+2+2}\right) G_{3}\left(x q^{4}\right) \\
+x^{6} q^{5+5+4+3+2+2} G_{3}\left(x q^{6}\right) .
\end{array}
$$

Now equation (5.15) may be viewed as an application of the Smallest Parts Decomposition Principle considering the partitions starting with parts $\leqq 2$. Thus to extend (5.15) to the full $G_{3}(x, y, q)$ we need only insert $y$ 's to account for appearances of evens an odd number of times. Hence

$$
\begin{aligned}
-\left(x^{3} q^{3+2+2}+x^{3} y q^{3+3+2}+x^{4} q^{3+3+2+2}+x^{4} y q^{3+3+2+1}+x^{4} y q^{4+3+2+2}\right) G_{3}\left(x q^{4}, y, q\right) \\
+x^{6} y q^{5+5+4+3+2+2} G_{3}\left(x q^{6}, y, q\right) .
\end{aligned}
$$

So if we define $E_{3}(x, q)$ to be the left-hand side of (5.16) minus the right-hand side, then we see that $E_{3}(x, q)$ is identically equal to 0 . Therefore

$$
\begin{aligned}
0= & E_{3}(x, q)-x^{2} y q^{5} E_{3}\left(x q^{2}, q\right) \\
= & G_{3}(x, y, q)-\left(1+q x(1+q y)\left(1+x q+x q^{3}\right)\right) G_{3}\left(x q^{2}, y, q\right) \\
& +\left(x^{2} y q^{5}+x^{3} q^{7}(1+q y)^{2}+x^{4} q^{9}\left(q+y+2 q^{2} y+y q^{4}+y^{2} q^{3}\right)\right) G_{3}\left(x q^{4}, y, q\right) \\
& -x^{5} q^{18} y(1+y q)\left(1+x q^{3}+x q^{5}\right) G_{3}\left(x q^{6}, y, q\right)+x^{8} y^{2} q^{38} G_{3}\left(x q^{8}, y, q\right) .
\end{aligned}
$$

Comparing (5.17) with (5.13) we see that the Defining $q$-Difference Equations Principle implies that

$$
G_{3}(x, y, q)=S_{3}(0,0 ; x ; q)
$$

\section{The Parity Indices}

The next few sections will be devoted to considerations of partitions subject to constraints on the following partition parameters.

Definition. Let $\lambda$ be a partition $\lambda: \lambda_{1}+\lambda_{2}+\cdots+\lambda_{j}$ where $\lambda_{1} \geqq \lambda_{2} \geqq \cdots \geqq \lambda_{j}$. We define $I_{U E}(\lambda)$ (resp. $I_{U O}$ ) to be the maximum length of nonincreasing subsequences of $\left\{\lambda_{1}, \lambda_{2}, \ldots, \lambda_{j}\right\}$ whose terms alternate in parity starting with an even (resp. odd) $\lambda_{i}$

Definition. Let $\lambda$ be a partition $\lambda: \lambda_{1}+\lambda_{2}+\cdots+\lambda_{j}$ where $\lambda_{1} \geqq \lambda_{2} \geqq \cdots \geqq \lambda_{j}$. We define $I_{L E}(\lambda)$ (resp. $I_{L O}$ ) to be the maximum length of nondecreasing subsequences 
of $\left\{\lambda_{1}, \lambda_{2}, \ldots, \lambda_{j}\right\}$ whose terms alternate in parity starting with an even (resp. odd) $\lambda_{i}$.

It is immediate from these definitions that if $\lambda_{1}$ is even, then $I_{U O}(\lambda)=I_{U E}(\lambda)-$ 1 , and if $\lambda_{1}$ is odd, then $I_{U E}(\lambda)=I_{U O}(\lambda)-1$. In the same way, if $\lambda_{j}$ is even, then $I_{L O}(\lambda)=I_{L E}(\lambda)-1$, and if $\lambda_{j}$ is odd, then $I_{L E}(\lambda)=I_{L O}(\lambda)-1$.

For example, if $\lambda=7+7+5+4+4+3+2+1+1$, then $I_{U E}(\lambda)=4, I_{U O}(\lambda)=5$, $I_{L E}(\lambda)=4, I_{L O}(\lambda)=5$.

\section{Lower Parity Indices in Partitions With Distinct Parts}

We begin with notation for the relevant partition functions:

$p_{e}(r, m, n)$ (resp. $\left.p_{o}(r, m, n)\right)$ is to denote the number of partitions of $n$ into $m$ distinct parts with lower even (resp. odd) parity index equal to $r$.

Next,

$$
P_{e}(y, x ; q)=\sum_{r, m, n \geqq 0} p_{e}(r, m, n) y^{r} x^{m} q^{n}
$$

and

$$
P_{o}(y, x ; q)=\sum_{r, m, n \geqq 0} p_{o}(r, m, n) y^{r} x^{m} q^{n} .
$$

\section{Theorem 7.}

$$
\begin{aligned}
P_{e}(y, x ; q) & =(1+x q) P_{o}(y, x q ; q), \\
P_{o}(y, x ; q) & =\sum_{n \geqq 0} \frac{x^{n} y^{n} q^{n(n+1) / 2}(-q / y)_{n}}{\left(q^{2} ; q^{2}\right)_{n}}, \\
& =(-x q)_{\infty} \sum_{n \geqq 0} \frac{(y)_{n}(-x q)^{n}}{\left(q^{2} ; q^{2}\right)_{n}} .
\end{aligned}
$$

Proof. By the Smallest Parts Decomposition Principle

$$
\begin{aligned}
& P_{e}(y, x ; q)=P_{o}(y, x q ; q)+x q P_{o}(y, x q ; q) \\
& P_{o}(y, x ; q)=P_{e}(y, x q ; q)+x q y P_{o}(y, x q ; q) .
\end{aligned}
$$

Equation (7.6) immediately establishes (7.3). We now substitute (7.6) into (7.7) to obtain

$$
P_{o}(y, x ; q)=\left(1+x q^{2}\right) P_{o}\left(y, x q^{2} ; q\right)+x q y P_{o}(y, x q ; q) .
$$

We now expand $P_{o}(y, x ; q)$ in a power series in $x$ :

$$
P_{o}(y, x ; q)=\sum_{n \geqq 0} A_{n} x^{n} .
$$

Substituting (7.9) into (7.8) and noting that $A_{0}=1$, we see that

$$
A_{n}=q^{2 n} A_{n}+q^{2 n} A_{n-1}+y q^{n} A_{n-1},
$$

or

$$
A_{n}=\frac{y q^{n}\left(1+q^{n} / y\right)}{\left(1-q^{2 n}\right)} A_{n-1} .
$$


Iteration then reveals that

$$
A_{n}=\frac{y^{n} q^{n(n+1) / 2}(-q / y)_{n}}{\left(q^{2} ; q^{2}\right)_{n}} .
$$

Combining (7.10) with (7.9), we see that (7.4) is established.

Finally by (7.4) using the basic hypergeometric series notation $[12 ; \mathrm{p} .4]$

(by $[12 ;$ p. 10, eq. (1.4.6)])

$$
\begin{aligned}
P_{o}(y, x ; q) & =\lim _{\tau \rightarrow 0} 2 \phi_{1}\left(\begin{array}{c}
-q / \tau,-q / y ; q, x y \tau \\
-q
\end{array}\right) \\
& =\lim _{\tau \rightarrow 0} \frac{(-x q)_{\infty}}{(x y \tau)_{\infty}}{ }_{2} \phi_{1}\left(\begin{array}{c}
\tau, y ; q,-x q \\
-q
\end{array}\right) \\
& =(-x q)_{\infty} \sum_{n=0}^{\infty} \frac{(y)_{n}(-x q)^{n}}{\left(q^{2} ; q^{2}\right)_{n}},
\end{aligned}
$$

which proves (7.5).

\section{Lower Parity Indices in Unrestricted Partitions}

Again we start with the relevant notation:

Let $u_{e}(r, m, n)$ (resp. $\left.u_{o}(r, m, n)\right)$ denote the number of partitions of $n$ into $m$ parts with lower even (resp. odd) parity index equal to $r$.

Next

$$
U_{e}(y, x ; q)=\sum_{r, m, n \geqq 0} u_{e}(r, m, n) y^{r} x^{m} q^{n}
$$

and

$$
U_{o}(y, x ; q)=\sum_{r, m, n \geqq 0} u_{o}(r, m, n) y^{r} x^{m} q^{n}
$$

\section{Theorem 8.}

$$
\begin{aligned}
U_{e}(y, x ; q) & =\frac{U_{o}(y, x q ; q)}{1-x q} \\
U_{o}(y, x ; q) & =\frac{1}{(x q)_{\infty}} \sum_{n \geqq 0} \frac{x^{n} y^{n} q^{n(n+1) / 2}(1 / y)_{n}}{\left(q^{2} ; q^{2}\right)_{n}}, \\
& =(1-x) \sum_{n \geqq 0} \frac{(-q y)_{n} x^{n}}{\left(q^{2} ; q^{2}\right)_{n}} .
\end{aligned}
$$

Proof. By the Smallest Parts Decomposition Principle

$$
\begin{aligned}
& U_{e}(y, x ; q)=U_{o}(y, x q ; q)+\frac{x q}{1-x q} U_{o}(y, x q ; q), \\
& U_{o}(y, x ; q)=U_{e}(y, x q ; q)+\frac{x q y}{1-x q} U_{o}(y, x q ; q) .
\end{aligned}
$$

Equation (8.6) immediately establishes (8.3). We now substitute (8.6) into (8.7) to obtain

$$
U_{o}(y, x ; q)=\frac{U_{o}\left(y, x q^{2} ; q\right)}{1-x q^{2}}+\frac{x q y}{1-x q} U_{o}(y, x q ; q) .
$$

Let us now define

$$
v(y, x ; q)=(x q)_{\infty} U_{o}(y, x ; q) .
$$


Substituting (8.9) into (8.8), we see that

$$
v(y, x ; q)=(1-x q) v\left(y, x q^{2} ; q\right)+x q y v(y, x q ; q) .
$$

We now expand $v(y, x ; q)$ in a power series in $x$ :

$$
v(y, x ; q)=\sum_{n \geqq 0} \alpha_{n} x^{n} .
$$

Substituting (8.11) in (8.10) and noting that $\alpha_{0}=1$, we see that

$$
\alpha_{n}=\alpha_{n} q^{2 n}-\alpha_{n-1} q^{2 n-1}+\alpha_{n-1} y q^{n},
$$

or

$$
\alpha_{n}=\frac{y q^{n}\left(1-q^{n-1} / y\right)}{\left(1-q^{2 n}\right)} \alpha_{n-1} .
$$

Iteration then reveals that

$$
\alpha_{n}=\frac{y^{n} q^{n(n+1) / 2}(1 / y)_{n}}{\left(q^{2} ; q^{2}\right)_{n}} .
$$

Combining (8.12) with (8.11) and (8.9), we see that (8.4) is established.

Finally by (8.4) using the notation of $[12 ;$ p. 4$]$

(by $[9 ;$ p. 10, eq. (1.4.6)])

$$
\begin{aligned}
U_{o}(y, x ; q) & =\frac{1}{(x q)_{\infty}} \lim _{\tau \rightarrow 0}{ }_{2} \phi_{1}\left(\begin{array}{c}
-q / \tau, 1 / y ; q, \tau x y \\
-q
\end{array}\right) \\
& =\frac{1}{(x q)_{\infty}} \lim _{\tau \rightarrow 0}(x)_{\infty 2} \phi_{1}\left(\begin{array}{c}
\tau,-q y ; q, x \\
-q
\end{array}\right) \\
& =(1-x) \sum_{n=0}^{\infty} \frac{(-q y)_{n} x^{n}}{\left(q^{2} ; q^{2}\right)_{n}}
\end{aligned}
$$

\section{Corollary 9.}

$$
U_{o}(y, x ; q)=\frac{1}{(x q)_{\infty}} P_{o}(-y q,-x / q ; q) .
$$

Proof. This follows immediately by comparison of (8.4) and (7.4).

\section{Upper Parity Indices in Partitions With Distinct Parts}

As before, we begin with notation for the relevant partition functions:

$\delta_{e}(N, r, m, n)$ (resp. $\left.\delta_{o}(N, r, m, n)\right)$ denotes the number of partitions of $n$ into $m$ distinct parts each $\leqq N$ and with upper even (resp. odd) parity index equal to $r$.

Next

$$
\begin{aligned}
& D_{e}(N, y, x ; q):=D_{e}(N)=\sum_{r, m, n \geqq 0} \delta_{e}(N, r, m, n) y^{r} x^{m} q^{n}, \\
& D_{o}(N, y, x ; q):=D_{o}(N)=\sum_{r, m, n \geqq 0} \delta_{o}(N, r, m, n) y^{r} x^{m} q^{n} .
\end{aligned}
$$


Theorem 10.

$$
\begin{aligned}
D_{o}(2 n-1)= & \sum_{i, j \geqq 0} x^{i} y^{2 j} q^{(i-j)^{2}+j^{2}+i+j}\left[\begin{array}{c}
n+j-1 \\
i
\end{array}\right]_{2}\left[\begin{array}{c}
i \\
2 j
\end{array}\right]_{1} \\
& +\sum_{i, j \geqq 0} x^{i} y^{2 j-1} q^{(i-j)^{2}+j^{2}+i-j}\left[\begin{array}{c}
n+j-1 \\
i
\end{array}\right]_{2}\left[\begin{array}{c}
i \\
2 j-1
\end{array}\right]_{1}, \\
D_{e}(2 n)= & \sum_{i, j \geqq 0} x^{i} y^{2 j} q^{(i-j)^{2}+j^{2}+j}\left[\begin{array}{c}
n+j \\
i
\end{array}\right]_{2}\left[\begin{array}{c}
i \\
2 j
\end{array}\right]_{1} \\
& +\sum_{i, j \geqq 0} x^{i} y^{2 j+1} q^{(i-j)^{2}+j^{2}+3 j+1}\left[\begin{array}{c}
n+j \\
i
\end{array}\right]_{2}\left[\begin{array}{c}
i \\
2 j+1
\end{array}\right]_{1} .
\end{aligned}
$$

Proof. By the Largest Part Decomposition Principle, we see that

$$
\begin{aligned}
D_{e}(2 N) & =D_{e}(2 N-1)+x y q^{2 N} D_{o}(2 N-1), \\
D_{o}(2 N) & =\left(1+x q^{2 N}\right) D_{o}(2 N-1), \\
D_{e}(2 N-1) & =\left(1+x q^{2 N-1}\right) D_{e}(2 N-2), \\
D_{o}(2 N-1) & =D_{o}(2 N-2)+x y q^{2 N-1} D_{e}(2 N-2) .
\end{aligned}
$$

In addition, we note the initial values $D_{e}(0)=1, D_{o}(1)=1+x y q$.

In order to prove our result we must reduce (9.5) - (9.8) to two recurrences only involving $D_{o}(2 N-1)$ and $D_{e}(2 N)$. By $(9.5)$ and $(9.7)$

$$
D_{e}(2 N)=\left(1+x q^{2 N-1}\right) D_{e}(2 N-2)+x y q^{2 N} D_{o}(2 N-1),
$$

and by (9.6) and (9.8)

$$
D_{o}(2 N-1)=\left(1+x q^{2 N-2}\right) D_{o}(2 N-3)+x y q^{2 N-1} D_{e}(2 N-2) .
$$

Clearly (9.9) and (9.10) plus the initial values completely define $D_{e}(2 N)$ and $D_{o}(2 N-1)$. To complete the proof of the theorem we need only show that the righthand sides of (9.3) and (9.4) satisfy the same recurrences. The initial conditions are clear by inspection.

Let us define $d_{o}(2 n-1)$ as the right-hand side of $(9.3)$ and $d_{e}(2 n)$ as the righthand side of (9.4).

First we note that

$$
\begin{aligned}
d_{e}(2 n)- & d_{e}(2 n-2) \\
= & \sum_{i, j \geqq 0} x^{i} y^{2 j} q^{(i-j)^{2}+j(j+1)} q^{2(n+j-i)}\left[\begin{array}{c}
n+j-1 \\
i-1
\end{array}\right]_{2}\left[\begin{array}{c}
i \\
2 j
\end{array}\right]_{1} \\
& +\sum_{i, j \geqq 0} x^{i} y^{2 j+1} q^{(i-j)^{2}+(j+1)(j+2)-1} q^{2(n+j-i)}\left[\begin{array}{c}
n+j-1 \\
i-1
\end{array}\right]_{2}\left[\begin{array}{c}
i \\
2 j+1
\end{array}\right]_{1} \\
(9.11)= & x q^{2 n-1}\left\{\sum_{i, j \geqq 0} x^{i} y^{2 j} q^{(i-j)^{2}+2(i-j)+j(j+1)+2 j-2 i}\left[\begin{array}{c}
n+j-1 \\
i
\end{array}\right]_{2}\left[\begin{array}{c}
i+1 \\
2 j
\end{array}\right]_{1}\right. \\
& \left.+\sum_{i, j \geqq 0} x^{i} y^{2 j+1} q^{(i-j)^{2}+2(i-j)+(j+1)(j+2)-1+2 j-2 i}\left[\begin{array}{c}
n+j-1 \\
i
\end{array}\right]_{2}\left[\begin{array}{c}
i+1 \\
2 j+1
\end{array}\right]_{1}\right\} .
\end{aligned}
$$


So to complete the proof of (9.9) with $D$ replaced by $d$, we need only identify $d_{e}(2 n-2)+y q d_{o}(2 n-1)$ with the expression inside the above curly brackets.

Now

$$
\begin{aligned}
d_{e}(2 n-2) & y q d_{o}(2 n-1) \\
= & \sum_{i, j \geqq 0} x^{i} y^{2 j} q^{(i-j)^{2}+j(j+1)}\left[\begin{array}{c}
n+j-1 \\
i
\end{array}\right]_{2}\left[\begin{array}{c}
i \\
2 j
\end{array}\right]_{1} \\
& +\sum_{i, j \geqq 0} x^{i} y^{2 j+1} q^{(i-j)^{2}+(j+1)(j+2)-1}\left[\begin{array}{c}
n+j-1 \\
i
\end{array}\right]_{2}\left[\begin{array}{c}
i \\
2 j+1
\end{array}\right]_{1} \\
& +\sum_{i, j \geqq 0} x^{i} y^{2 j+1} q^{i^{2}-(2 j-1) i+\left(\begin{array}{c}
2 j+1 \\
2
\end{array}\right)+1}\left[\begin{array}{c}
n+j-1 \\
i
\end{array}\right]_{2}\left[\begin{array}{c}
i \\
2 j
\end{array}\right]_{1} \\
& +\sum_{i, j \geqq 0} x^{i} y^{2 j} q^{i^{2}-(2 j-1) i+\left(\begin{array}{c}
2 j \\
2
\end{array}\right)+1}\left[\begin{array}{r}
n+j-1 \\
i
\end{array}\right]_{2}\left[\begin{array}{c}
i \\
2 j-1
\end{array}\right]_{1} \\
= & \left.\sum_{i, j \geqq 0} x^{i} y^{2 j} q^{i^{2}-2 i j+2 j^{2}+j}\left[\begin{array}{r}
n+j-1 \\
i
\end{array}\right]_{2}\left(\begin{array}{c}
i \\
2 j
\end{array}\right]_{1}+q^{i-2 j+1}\left[\begin{array}{c}
i \\
2 j-1
\end{array}\right]_{1}\right) \\
& +\sum_{i, j \geqq 0} x^{i} y^{2 j+1} q^{i^{2}-2 i j+2 j^{2}+3 j+1}\left[\begin{array}{c}
n+j-1 \\
i
\end{array}\right]_{2}\left(\left[\begin{array}{c}
i \\
2 j+1
\end{array}\right]_{1}+q^{i-2 j}\left[\begin{array}{c}
i \\
2 j
\end{array}\right]_{1}\right)
\end{aligned}
$$

(where we have combined the first with the fourth sum and the second with the third)

(by $(2.12)$ )

$$
\begin{aligned}
= & \sum_{i, j \geqq 0} x^{i} y^{2 j} q^{i^{2}-2 i j+2 j^{2}+j}\left[\begin{array}{c}
n+j-1 \\
i
\end{array}\right]_{2}\left[\begin{array}{c}
i+1 \\
2 j
\end{array}\right]_{1} \\
& +\sum_{i, j \geqq 0} x^{i} y^{2 j+1} q^{i^{2}-2 i j+2 j^{2}+3 j+1}\left[\begin{array}{c}
n+j-1 \\
i
\end{array}\right]_{2}\left[\begin{array}{c}
i+1 \\
2 j+1
\end{array}\right]_{1} .
\end{aligned}
$$

Now inspection reveals that this last entry in (9.12) is identical with the final expression in curly brackets in (9.11). Hence (9.9) has been established with the $D$ 's replaced by the $d$ 's.

To conclude we must do the same for (9.10). Now we see that

$$
\begin{aligned}
& d_{o}(2 n-1)-d_{o}(2 n-3) \\
& =\sum_{i, j \geqq 0} x^{i} y^{2 j} q^{i^{2}-(2 j-1) i+\left(\begin{array}{c}
2 j+1 \\
2
\end{array}\right)} q^{2(n+j-1-i)}\left[\begin{array}{c}
n+j-2 \\
i-1
\end{array}\right]_{2}\left[\begin{array}{c}
i \\
2 j
\end{array}\right]_{1} \\
& +\sum_{i, j \geqq 0} x^{i} y^{2 j-1} q^{i^{2}-(2 j-1) i+\left(\begin{array}{c}
2 j \\
2
\end{array}\right)} q^{2(n+j-1-i)}\left[\begin{array}{c}
n+j-2 \\
i-1
\end{array}\right]_{2}\left[\begin{array}{c}
i \\
2 j-1
\end{array}\right]_{1} \\
& =x q^{2 n-2}\left\{\sum_{i, j \geqq 0} x^{i} y^{2 j} q^{i^{2}-(2 j-1) i+\left(\begin{array}{c}
2 j+1 \\
2
\end{array}\right)}\left[\begin{array}{c}
n+j-2 \\
i
\end{array}\right]_{2}\left[\begin{array}{c}
i+1 \\
2 j
\end{array}\right]_{1}\right. \\
& \left.+\sum_{i, j \geqq 0} x^{i} y^{2 j-1} q^{i^{2}-(2 j-1) i+\left(\begin{array}{c}
2 j \\
2
\end{array}\right)}\left[\begin{array}{c}
n+j-2 \\
i
\end{array}\right]_{2}\left[\begin{array}{c}
i+1 \\
2 j-1
\end{array}\right]_{1}\right\} \text {, }
\end{aligned}
$$


where in the final line we have shifted $i$ to $i+1$ in each sum.

So to finish the proof of (9.10) with $D$ replaced by $d$, we need only identify $d_{o}(2 n-3)+y q d_{e}(2 n-2)$ with the above expression contained in the curly brackets.

$$
\begin{aligned}
& d_{o}(2 n-3)+y q d_{e}(2 n-2) \\
& =\sum_{i, j \geqq 0} x^{i} y^{2 j} q^{i^{2}-(2 j-1) i+\left(\begin{array}{c}
2 j+1 \\
2
\end{array}\right)}\left[\begin{array}{c}
n+j-2 \\
i
\end{array}\right]_{2}\left[\begin{array}{c}
i \\
2 j
\end{array}\right]_{1} \\
& +\sum_{i, j \geqq 0} x^{i} y^{2 j-1} q^{i^{2}-(2 j-1) i+\left(\begin{array}{c}
2 j \\
2
\end{array}\right)}\left[\begin{array}{c}
n+j-2 \\
i
\end{array}\right]_{2}\left[\begin{array}{c}
i \\
2 j-1
\end{array}\right]_{1} \\
& +\sum_{i, j \geqq 0} x^{i} y^{2 j+1} q^{(i-j)^{2}+j(j+1)+1}\left[\begin{array}{c}
n+j-1 \\
i
\end{array}\right]_{2}\left[\begin{array}{c}
i \\
2 j
\end{array}\right]_{1} \\
& +\sum_{i, j \geqq 0} x^{i} y^{2 j+2} q^{(i-j)^{2}+(j+1)(j+2)}\left[\begin{array}{c}
n+j-1 \\
i
\end{array}\right]_{2}\left[\begin{array}{c}
i \\
2 j+1
\end{array}\right]_{1} \\
& =\sum_{i, j \geqq 0} x^{i} y^{2 j} q^{i^{2}-(2 j-1) i+\left(\begin{array}{c}
2 j+1 \\
2
\end{array}\right)}\left[\begin{array}{c}
n+j-2 \\
i
\end{array}\right]_{2}\left(\left[\begin{array}{c}
i \\
2 j
\end{array}\right]_{1}+q^{i-2 j+1}\left[\begin{array}{c}
i \\
2 j-1
\end{array}\right]_{1}\right) \\
& +\sum_{i, j \geqq 0} x^{i} y^{2 j-1} q^{i^{2}-(2 j-1)+\left(\begin{array}{c}
2 j \\
2
\end{array}\right)}\left[\begin{array}{c}
n+j-2 \\
i
\end{array}\right]_{2}\left(\left[\begin{array}{c}
i \\
2 j-1
\end{array}\right]_{1}+q^{i-2 j+2}\left[\begin{array}{c}
i \\
2 j-2
\end{array}\right]_{1}\right)
\end{aligned}
$$

(where we replaced $j$ by $j-1$ in the third and fourth sums and then combined sum 1 with sum 4 and sum 2 with sum 3 )

(by $(2.12)$ )

$$
=\sum_{i, j \geqq 0} x^{i} y^{2 j} q^{i^{2}-(2 j-1) i+\left(\begin{array}{c}
2 j+1 \\
2
\end{array}\right)}\left[\begin{array}{c}
n+j-2 \\
i
\end{array}\right]_{2}\left[\begin{array}{c}
i+1 \\
2 j
\end{array}\right]_{1}
$$

$$
+\sum_{i, j \geqq 0} x^{i} y^{2 j-1} q^{i^{2}-(2 j-1) i+\left(\begin{array}{c}
2 j \\
2
\end{array}\right)}\left[\begin{array}{c}
n+j-2 \\
i
\end{array}\right]_{2}\left[\begin{array}{c}
i+1 \\
2 j-1
\end{array}\right]_{1} .
$$

As before, the last entry in (9.14) is identical with the final expression in curly brackets in (9.13). Hence (9.10) is established with $D$ replaced by $d$. Thus our theorem is proved.

\section{Corollary 11.}

$$
\begin{aligned}
& D_{o}(\infty)=\sum_{i, j \geqq 0} \frac{x^{i} y^{2 j} q^{(i-j)^{2}+j^{2}+i+j}}{(-q ; q)_{i}(q)_{2 j}(q)_{i-2 j}}+\sum_{i, j \geqq 0} \frac{x^{i} y^{2 j-1} q^{(i-j)^{2}+j^{2}+i-j}}{(-q ; q)_{i}(q)_{2 j-1}(q)_{i-2 j+1}} \\
& D_{e}(\infty)=\sum_{i, j \geqq 0} \frac{x^{i} y^{2 j} q^{(i-j)^{2}+j^{2}+j}}{(-q ; q)_{i}(q)_{2 j}(q)_{i-2 j}}+\sum_{i, j \geqq 0} \frac{x^{i} y^{2 j+1} q^{(i-j)^{2}+j^{2}+3 j+1}}{(-q ; q)_{i}(q)_{2 j+1}(q)_{i-2 j-1}} .
\end{aligned}
$$

Proof. Let $N \rightarrow \infty$ in the theorem and then algebraically simplify each term.

We note that if we set $y=0$ in (9.16), then $D_{e}(\infty)$ must be the generating function for partitions into distinct odd parts, and in this instance the right-hand 
side of (9.16) reduces to the familiar sum in Euler's identity

$$
\sum_{i \geqq 0} \frac{x^{i} q^{i^{2}}}{\left(q^{2} ; q^{2}\right)_{i}}=\left(-x q ; q^{2}\right)_{\infty} .
$$

(cf. $[12 ;$ p. 9 , eq. (1.3.16)]).

Also if we set $y=0$ in (9.15) then $D_{o}(\infty)$ must be the generating function for partitions into distinct even parts, and in this instance the right-hand side of (9.15) reduces to a change of $x$ to $x q$ in (9.17).

However, setting $y=1$ in either $D_{o}(\infty)$ or $D_{e}(\infty)$ must produce the generating function for all partitions into distinct parts. Consequently

$$
\begin{aligned}
(-x q ; q)_{\infty} & =\sum_{i, j \geqq 0} \frac{x^{i} q^{(i-j)^{2}+j^{2}+i+j}}{(-q ; q)_{i}(q)_{2 j}(q)_{i-2 j}}+\sum_{i, j \geqq 0} \frac{x^{i} q^{(i-j)^{2}+j^{2}+i-j}}{(-q ; q)_{i}(q)_{2 j-1}(q)_{i-2 j+1}} \\
& =\sum_{i, j \geqq 0} \frac{x^{i} q^{(i-j)^{2}+j^{2}+j}}{(-q ; q)_{i}(q)_{2 j}(q)_{i-2 j}}+\sum_{i, j \geqq 0} \frac{x^{i} q^{(i-j)^{2}+j^{2}+3 j+1}}{(-q ; q)_{i}(q)_{2 j+1}(q)_{i-2 j-1}}
\end{aligned}
$$

which appear to be new.

We note that similar finite results can be obtained by setting $y=0$ and $y=1$ in Theorem 10. The $y=0$ case is the classical $q$-binomial theorem $[7 ;$ p. 36 , eq. (3.3.6)]. The $y=1$ case also appears to be new.

\section{Upper Parity Indices With UnRestricted Parts}

Again we begin with notation for the relevant partition functions:

$\phi_{e}(N, r, m, n)$ (resp. $\left.\phi_{o}(N, r, m, n)\right)$ denotes the number of partitions of $n$ into $m$ parts each $\leqq N$ and with upper even (resp. odd) parity index equal to $r$.

Next

$$
F_{e}(N, y, x ; q):=F_{e}(N)=\sum_{r, m, n \geqq 0} \phi_{e}(N, r, m, n) y^{r} x^{m} q^{n}
$$

and

$$
F_{o}(N, y, x ; q):=F_{o}(N)=\sum_{r, m, n \geqq 0} \phi_{o}(N, r, m, n) y^{r} x^{m} q^{n}
$$

Theorem 12.

$$
\begin{aligned}
F_{e}(2 n-1)= & \sum_{i, j \geqq 0} x^{i} y^{2 j} q^{\left(\begin{array}{c}
2 j \\
2
\end{array}\right)+i}\left[\begin{array}{c}
n+i-j-1 \\
i
\end{array}\right]_{2}\left[\begin{array}{c}
i \\
2 j
\end{array}\right]_{1} \\
& +\sum_{i, j \geqq 0} x^{i} y^{2 j+1} q^{\left(\begin{array}{c}
2 j+1 \\
2
\end{array}\right)+2 i}\left[\begin{array}{c}
n+i-j-2 \\
i
\end{array}\right]_{2}\left[\begin{array}{c}
i \\
2 j+1
\end{array}\right]_{1}, \\
F_{o}(2 n)= & \sum_{i, j \geqq 0} x^{i} y^{2 j} q^{\left(\begin{array}{c}
2 j \\
2
\end{array}\right)+2 i}\left[\begin{array}{c}
n+i-j-1 \\
i
\end{array}\right]_{2}\left[\begin{array}{c}
i \\
2 j
\end{array}\right]_{1} \\
& +\sum_{i, j \geqq 0} x^{i} y^{2 j+1} q^{\left(\begin{array}{c}
2 j+1 \\
2
\end{array}\right)+i}\left[\begin{array}{c}
n+i-j-1 \\
i
\end{array}\right]_{2}\left[\begin{array}{c}
i \\
2 j+1
\end{array}\right]_{1} .
\end{aligned}
$$


Proof. By the Largest Part Decomposition Principle, we see that

$$
\begin{aligned}
F_{e}(2 n) & =F_{e}(2 n-1)+x y q^{2 n} F_{o}(2 n), \\
F_{o}(2 n) & =F_{o}(2 n-1)+x q^{2 n} F_{o}(2 n), \\
F_{e}(2 n-1) & =F_{e}(2 n-2)+x q^{2 n-1} F_{e}(2 n-1), \\
F_{o}(2 n-1) & =F_{o}(2 n-2)+x y q^{2 n-1} F_{e}(2 n-1) .
\end{aligned}
$$

Now we eliminate $F_{o}(2 n-1)$ and $F_{e}(2 n)$ from $(10.5)-(10.8)$. This yields

$$
F_{e}(2 n-1)=F_{e}(2 n-3)+x y q^{2 n-2} F_{o}(2 n-2)+x q^{2 n-1} F_{e}(2 n-1),
$$

and

$$
F_{o}(2 n)=F_{o}(2 n-2)+x y q^{2 n-1} F_{e}(2 n-1)+x q^{2 n} F_{o}(2 n) .
$$

At first glance, it would appear that these are not defining recurrences for the $F$ 's in that the left-hand side of each equation also appears on the right. However, the offending expression on the right can easily be moved to the left to overcome this objection. Of course, this means that the $F^{\prime} s$ will be rational functions not polynomials as were the $D$ 's of Section 9 .

Thus (10.9) and (10.10) together with the initial values $F_{o}(0)=1$ and $F_{e}(1)=$ $\frac{1}{1-x q}$ uniquely determine $F_{o}(2 n)$ and $F_{e}(2 n-1)$. Inspection reveals that for $n=0$, the right-hand side of $(10.4)=1$, and for $n=1$, the right-hand side of (10.3) is

$$
\sum_{i \geqq 0} x^{i} q^{i}=\frac{1}{1-x q}
$$

Consequently in order to complete the proof of our theorem we need only show that the right-hand sides of (10.3) and (10.4) satisfy (10.9) and (10.10).

Let us define $f_{e}(2 n-1)$ as the right-hand side of $(10.3)$ and $f_{o}(2 n)$ as the righthand side of (10.4).

We treat (10.9) first noting that

$$
\begin{aligned}
& f_{e}(2 n-1)-f_{e}(2 n-3) \\
& =\sum_{i, j \geqq 0} x^{i} y^{2 j} q^{\left(\begin{array}{c}
2 j \\
2
\end{array}\right)+i}\left[\begin{array}{c}
n+i-j-2 \\
i-1
\end{array}\right]_{2} q^{2(n-j-1)}\left[\begin{array}{c}
i \\
2 j
\end{array}\right]_{1} \\
& \left.+\sum_{i, j \geqq 0} x^{i} y^{2 j+1} q^{(2 j+1}\right)+2 i\left[\begin{array}{c}
n+i-j-3 \\
i-1
\end{array}\right]_{2} q^{2(n-j-2)}\left[\begin{array}{c}
i \\
2 j+1
\end{array}\right]_{1} \\
& =x q^{2 n-2}\left\{\sum_{i, j \geqq 0} x^{i} y^{2 j} q^{(2 j-1)+i}\left[\begin{array}{c}
n+i-j-1 \\
i
\end{array}\right]_{2}\left[\begin{array}{c}
i+1 \\
2 j
\end{array}\right]_{1}\right. \\
& \left.+\sum_{i, j \geqq 0} x^{i} y^{2 j+1} q^{\left(\begin{array}{c}
2 j \\
2
\end{array}\right)+2 i}\left[\begin{array}{c}
n+i-j-2 \\
i
\end{array}\right]_{2}\left[\begin{array}{c}
i+1 \\
2 j+1
\end{array}\right]_{1}\right\} \text {. }
\end{aligned}
$$


Therefore to conclude our treatment of $(10.9)$ we must show that $y f_{o}(2 n-2)+$ $q f_{e}(2 n-1)$ is equal to this last expression in curly brackets.

$$
\begin{aligned}
y f_{o}(2 n-2) & +q f_{e}(2 n-1) \\
= & \sum_{i, j \geqq 0} x^{i} y^{2 j+1}\left[\begin{array}{c}
n+i-j-2 \\
i
\end{array}\right]_{2}\left[\begin{array}{c}
i \\
2 j
\end{array}\right]_{1} q^{2 i+\left(\begin{array}{c}
2 j \\
2
\end{array}\right)} \\
& +\sum_{i, j \geqq 0} x^{i} y^{2 j+2}\left[\begin{array}{c}
n+i-j-2 \\
i
\end{array}\right]_{2}\left[\begin{array}{c}
i \\
2 j+1
\end{array}\right]_{1} q^{i+\left(\begin{array}{c}
2 j+1 \\
2
\end{array}\right)} \\
& +\sum_{i, j \geqq 0} x^{i} y^{2 j} q^{\left(\begin{array}{c}
2 j \\
2
\end{array}\right)+i+1}\left[\begin{array}{c}
n+i-j-1 \\
i
\end{array}\right]_{2}\left[\begin{array}{c}
i \\
2 j
\end{array}\right]_{1} \\
& +\sum_{i, j \geqq 0} x^{i} y^{2 j+1} q^{\left(\begin{array}{c}
2 j+1 \\
2
\end{array}\right)+2 i+1}\left[\begin{array}{c}
n+i-j-2 \\
i
\end{array}\right]_{2}\left[\begin{array}{c}
i \\
2 j+1
\end{array}\right]_{1} \\
= & \sum_{i, j \geqq 0} x^{i} y^{2 j}\left[\begin{array}{c}
n+i-j-1 \\
i
\end{array}\right]_{2} q^{i+\left(\begin{array}{c}
2 j-1 \\
2
\end{array}\right)}\left(\left[\begin{array}{c}
i \\
2 j-1
\end{array}\right]_{1}+q^{2 j}\left[\begin{array}{c}
i \\
2 j
\end{array}\right]_{1}\right) \\
& +\sum_{i, j \geqq 0} x^{i} y^{2 j+1}\left[\begin{array}{c}
n+i-j-2 \\
i
\end{array}\right]_{2} q^{2 i+\left(\begin{array}{c}
2 j \\
2
\end{array}\right)}\left(\left[\begin{array}{c}
i \\
2 j
\end{array}\right]_{1}+q^{2 j+1}\left[\begin{array}{c}
i \\
2 j+1
\end{array}\right]_{1}\right)
\end{aligned}
$$

(where we have combined the second and third sums into the new first sum and the first and fourth in to the new second sum)

$($ by $(2.11))=\sum_{i, j \geqq 0} x^{i} y^{2 j} q^{i+\left(\begin{array}{c}2 j-1 \\ 2\end{array}\right)}\left[\begin{array}{c}n+i-j-1 \\ i\end{array}\right]_{2}\left[\begin{array}{c}i+1 \\ 2 j\end{array}\right]_{1}$

$$
+\sum_{i, j \geqq 0} x^{i} y^{2 j+1} q^{2 i+\left(\begin{array}{c}
2 j \\
2
\end{array}\right)}\left[\begin{array}{c}
n+i-j-2 \\
i
\end{array}\right]_{2}\left[\begin{array}{c}
i \\
2 j+1
\end{array}\right]_{1} \text {, }
$$

which is precisely the expression inside the curly brackets in the final entry in (10.11).

We now move to (10.10) for the $f$ 's noting that

$$
\begin{aligned}
& f_{o}(2 n)-f_{o}(2 n-2) \\
& =\sum_{i, j \geqq 0} x^{i} y^{2 j}\left[\begin{array}{c}
n+i-j-2 \\
i-1
\end{array}\right]_{2} q^{2(n-j-1)}\left[\begin{array}{c}
i \\
2 j
\end{array}\right]_{1} q^{2 i+\left(\begin{array}{c}
2 j \\
2
\end{array}\right)} \\
& +\sum_{i, j \geqq 0} x^{i} y^{2 j+1}\left[\begin{array}{c}
n+i-j-2 \\
i-1
\end{array}\right]_{2} q^{2(n-j-1)}\left[\begin{array}{c}
i \\
2 j+1
\end{array}\right]_{1} q^{i+\left(\begin{array}{c}
2 j+1 \\
2
\end{array}\right)} \\
& =x q^{2 n-1}\left\{\sum_{i, j \geqq 0} x^{i} y^{2 j}\left[\begin{array}{c}
n+i-j-1 \\
i
\end{array}\right]_{2}\left[\begin{array}{c}
i+1 \\
2 j
\end{array}\right]_{1} q^{\left(\begin{array}{c}
2 j-1 \\
2
\end{array}\right)+2 i}\right. \\
& \left.+\sum_{i, j \geqq 0} x^{i} y^{2 j+1}\left[\begin{array}{c}
n+i-j-1 \\
i
\end{array}\right]_{2}\left[\begin{array}{c}
i+1 \\
2 j+1
\end{array}\right]_{1} q^{\left(\begin{array}{c}
2 j \\
2
\end{array}\right)+i}\right\} .
\end{aligned}
$$


In order to complete (10.10) for the $f$ 's, we must identify this last expression in curly brackets with $y f_{e}(2 n-1)+q f_{o}(2 n)$.

$$
\begin{aligned}
& \left.y f_{e}(2 n-1)\right)+q f_{o}(2 n) \\
& =\sum_{i, j \geqq 0} x^{i} y^{2 j+1} q^{\left(\begin{array}{c}
2 j \\
2
\end{array}\right)+i}\left[\begin{array}{c}
n+i-j-1 \\
i
\end{array}\right]_{2}\left[\begin{array}{c}
i \\
2 j
\end{array}\right]_{1} \\
& \left.+\sum_{i, j \geqq 0} x^{i} y^{2 j+2} q^{(2 j+1}\right)+2 i\left[\begin{array}{c}
n+i-j-2 \\
i
\end{array}\right]_{2}\left[\begin{array}{c}
i \\
2 j+1
\end{array}\right]_{1} \\
& +\sum_{i, j \geqq 0} x^{i} y^{2 j} q^{2 i+\left(\begin{array}{c}
2 j \\
2
\end{array}\right)+1}\left[\begin{array}{c}
n+i-j-1 \\
i
\end{array}\right]_{2}\left[\begin{array}{c}
i \\
2 j
\end{array}\right]_{1} \\
& +\sum_{i, j \geqq 0} x^{i} y^{2 j+1} q^{i+\left(\begin{array}{c}
2 j+1 \\
2
\end{array}\right)+1}\left[\begin{array}{c}
n+i-j-1 \\
i
\end{array}\right]_{2}\left[\begin{array}{c}
i \\
2 j+1
\end{array}\right]_{1}
\end{aligned}
$$

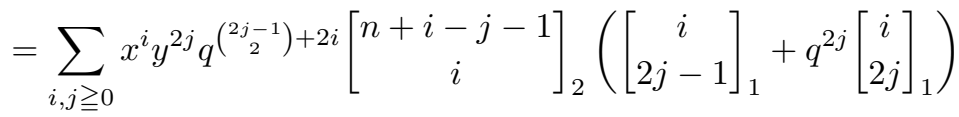

$$
\begin{aligned}
& +\sum_{i, j \geqq 0} x^{i} y^{2 j+1} q^{\left(\begin{array}{c}
2 j \\
2
\end{array}\right)+i}\left[\begin{array}{c}
n+i-j-1 \\
i
\end{array}\right]_{2}\left(\left[\begin{array}{c}
i \\
2 j
\end{array}\right]_{1}+q^{2 j+1}\left[\begin{array}{c}
i \\
2 j+1
\end{array}\right]_{1}\right)
\end{aligned}
$$

(combining the second and third sums into the first new sum, and the first and fourth into the second new sum)

$($ by $\left.(2.11))=\sum_{i, j \geqq 0} x^{i} y^{2 j} q^{(2 j-1}\right)+2 i\left[\begin{array}{c}n+i-j-1 \\ i\end{array}\right]_{2}\left[\begin{array}{c}i+1 \\ 2 j\end{array}\right]_{1}$

$$
+\sum_{i, j \geqq 0} x^{i} y^{2 j+1} q^{\left(\begin{array}{c}
2 j \\
2
\end{array}\right)+i}\left[\begin{array}{c}
n+i-j-1 \\
i
\end{array}\right]_{2}\left[\begin{array}{c}
i+1 \\
2 j+1
\end{array}\right]_{1},
$$

and this is indeed the expression inside curly brackets in (10.13). So (10.10) is proved for the f's, and, as a result, our theorem is proved.

\section{Corollary 13.}

$$
\begin{aligned}
& F_{e}(\infty)=\sum_{i, j \geqq 0} \frac{x^{i} y^{2 j} q^{\left(\begin{array}{c}
2 j \\
2 j
\end{array}\right)+i}}{(-q ; q)_{i}(q)_{2 j}(q)_{i-2 j}}+\sum_{i, j \geqq 0} \frac{\left.x^{i} y^{2 j+1} q^{(2 j+1}\right)+2 i}{(-q ; q)_{i}(q)_{2 j+1}(q)_{i-2 j-1}}, \\
& F_{o}(\infty)=\sum_{i, j \geqq 0} \frac{x^{i} y^{2 j} q^{\left(\begin{array}{c}
2 j \\
2
\end{array}\right)+2 i}}{(-q ; q)_{i}(q)_{2 j}(q)_{i-2 j}}+\sum_{i, j \geqq 0} \frac{\left.x^{i} y^{2 j+1} q^{(2 j+1}\right)+i}{(-q ; q)_{i}(q)_{2 j+1}(q)_{i-2 j-1}} .
\end{aligned}
$$

Proof. Let $n \rightarrow \infty$ in (10.3) and (10.4) respectively.

We note that setting $y=0$ in (10.15) yields the generating function for partitions into odd parts (cf. [12; p. 9, eq. (1.3.15)])

$$
\sum_{i \geqq 0} \frac{x^{i} q^{i}}{\left(q^{2} ; q^{2}\right)_{i}}=\frac{1}{\left(x q ; q^{2}\right)_{\infty}},
$$


while setting $y=0$ in (10.16) yields the generating function for partitions into even parts

$$
\sum_{i \geqq 0} \frac{x^{i} q^{2 i}}{\left(q^{2} ; q^{2}\right)_{i}}=\frac{1}{\left(x q^{2} ; q^{2}\right)_{\infty}} .
$$

Identities (10.17) and (10.18) both go back to Euler.

However setting $y=1$ in either $F_{e}(\infty)$ or $F_{o}(\infty)$ must produce the generating function for all partitions.

$$
\begin{aligned}
\frac{1}{(x q)_{\infty}} & =\sum_{i, j \geqq 0} \frac{x^{i} q^{\left(\begin{array}{c}
2 j \\
2
\end{array}\right)+i}}{(-q ; q)_{i}(q)_{2 j}(q)_{i-2 j}}+\sum_{i, j \geqq 0} \frac{x^{i} q^{\left(\begin{array}{c}
2 j+1 \\
2
\end{array}\right)+2 i}}{(-q ; q)_{i}(q)_{2 j+1}(q)_{i-2 j-1}} \\
& =\sum_{i, j \geqq 0} \frac{x^{i} q^{\left(\begin{array}{c}
(j) \\
2
\end{array}\right)+2 i}}{(-q ; q)_{i}(q)_{2 j}(q)_{i-2 j}}+\sum_{i, j \geqq 0} \frac{\left.x^{i} q^{(2 j+1}\right)+i}{(-q ; q)_{i}(q)_{2 j+1}(q)_{i-2 j-1}}
\end{aligned}
$$

which appear to be new.

We note that, as in Section 9, similar finite results can be obtained by setting $y=0$ and $y=1$ in Theorem 12. The $y=0$ case reduces to instances of the classical $q$-binomial series [7; p. 36, eq. (3.3.7)]. The $y=1$ case again appears to be new.

\section{Part Size And Parity Indices}

The previous two sections were devoted to considerations of partitions enumerated according to parity indices. Surprisingly, when we connect part size to a parity index, we come upon a new interpretation of the Rogers-Ramanujan identities.

Definition. We say that a partition $\lambda$ has even (resp. odd) ample part size if each part $\lambda_{i}$ is larger than $I_{U E}(\lambda)$ (resp. $I_{U O}(\lambda)$ ).

Let $R_{e}(N, r, m, n)$ denote the number of partitions of $n$ into $m$ distinct parts each $\leqq 2 N$; in addition, we require even ample part size with upper even parity index $=r$.

Next

$$
\rho_{e}(N, y, x, q)=\sum_{r, m, n \geqq 0} R_{e}(N, r, m, n) y^{r} x^{m} q^{n} .
$$

Theorem 14.

$$
\rho_{e}(N, y, x, q)=\sum_{j=0}^{N}\left[\begin{array}{c}
N \\
j
\end{array}\right]_{q^{2}} q^{j^{2}} x^{i}(-y q)_{j} .
$$

Proof. We note by the Largest Part Decomposition Principle, that

$$
\rho_{e}(N, y, x, q)=\left(1+x q^{2 N-1}\right) \rho_{e}(N-1, y, x, q)+x y q^{2 N} \rho_{e}(N-1, y, x q, q)
$$

The last term in the above by accounts for those partitions in which $2 N$ appears; the $x q$ in $\rho_{e}(N-1, y, x q, q)$ both increases the part size by 1 (which is necessary because the $x y q^{2 N}$ out front accounts for an increase in the upper even parity index) and changes the upper even index to the upper odd index to which $2 N$ is attached to recover the upper even index.

In addition, we see that

$$
\rho_{e}(0, y, x, q)=1
$$


We observe that (11.3) and (11.4) completely determine $\rho_{e}(N, y, x, q)$.

Now we consider

$$
\bar{\rho}(N, y, x, q)=\sum_{j=0}^{N}\left[\begin{array}{c}
N \\
j
\end{array}\right]_{q^{2}} q^{j^{2}} z^{j}(-y q)_{j} .
$$

$\bar{\rho}(N, y, x, q)-\bar{\rho}(N-1, y, x, q)$

$$
=\sum_{j \geqq 0}\left(\left[\begin{array}{c}
N \\
j
\end{array}\right]_{q^{2}}-\left[\begin{array}{c}
N-1 \\
j
\end{array}\right]_{q^{2}}\right) q^{j^{2}} x^{j}(-y q)_{j}
$$

$$
\begin{aligned}
& =\sum_{j \geqq 0} q^{2(N-j)}\left[\begin{array}{c}
N-1 \\
j-1
\end{array}\right]_{q^{2}} q^{j^{2}} x^{j}(-y q)_{j} \\
& =x q^{2 N-1} \sum_{j \geqq 0}\left[\begin{array}{c}
N-1 \\
j
\end{array}\right]_{q^{2}} q^{j^{2}} x^{j}(-y q)_{j}\left(1+y q^{j+1}\right) \\
& =x q^{2 N-1} \bar{\rho}(N-1, y, x, q)+x y q^{2 N} \sum_{j \geqq 0}\left[\begin{array}{c}
N-1 \\
j
\end{array}\right]_{q^{2}} q^{j^{2}}(x q)^{j}(-y q)_{j} \\
& =x q^{2 N-1} \bar{\rho}(N-1, y, x, q)+x y q^{2 N} \bar{\rho}(N-1, y, x q, q) .
\end{aligned}
$$

Thus in light of the fact that $\bar{\rho}(0, y, x, q)=\rho_{e}(0, y, x, q)$ and that (11.3) and (11.6) are identical recurrences, we see by mathematical induction that for each $N \geqq 0$

$$
\rho_{e}(N, y, x, q)=\bar{\rho}(N, y, x, q),
$$

and this proves our theorem.

It is now a straightforward matter to connect partitions into distinct parts of ample size with the Rogers-Ramanujan identities [7; p. 113].

Corollary 15. The number of partitions of $n$ into distinct parts of even ample size equals the number of partitions of $n$ into parts $\equiv \pm 1(\bmod 5)$.

Proof. Setting $x=y=1$ in (11.2) and letting $N \rightarrow \infty$, we see that the generating function for partitions into distinct parts with even ample part size is

(by [4; p. 113, Cor. 7.9]) $\sum_{j \geqq 0} \frac{q^{j^{2}}}{\left(q^{2} ; q^{2}\right)_{j}}(-q)_{j}=\sum_{j \geqq 0} \frac{q^{j^{2}}}{(q)_{j}}=\frac{1}{\left(q ; q^{5}\right)_{\infty}\left(q^{4} ; q^{5}\right)_{\infty}}$,

and the infinite product expression is just the generating function for partitions with parts $\equiv \pm 1(\bmod 5)$.

It is also easy to obtain a similar interpretation for the second Rogers-Ramanujan identity.

Corollary 16. The number of partitions of $n$ into distinct parts of odd ample size equals the number of partitions of $n$ into parts $\equiv \pm 2(\bmod 5)$.

Proof. If we replace $x$ by $x q$ in (11.1), we see that the exponent on $y$ now measures the odd upper index. Thus setting $x=q, y=1$ in (11.2) and letting $N \rightarrow \infty$, we 
see that the generating function for partitions into distinct parts with odd ample part size is

(by [7; p. 113, Cor. 7.10]) $\quad \sum_{j \geqq 0} \frac{q^{j^{2}+j}(-q)_{j}}{\left(q^{2} ; q^{2}\right)_{j}}=\sum_{j \geqq 0} \frac{q^{j^{2}+j}}{(q)_{j}}=\frac{1}{\left(q^{2} ; q^{5}\right)_{\infty}\left(q^{3} ; q^{5}\right)_{\infty}}$,

and the infinite product expression is just the generating function for partitions with parts $\equiv \pm 2(\bmod 5)$.

\section{Mock Theta Functions and Little $q$-Jacobi Polynomials}

In the last section we saw that

$$
\rho_{e}(\infty, y, x, q)=\sum_{N \geqq 0} \frac{q^{n^{2}} x^{n}(-y q ; q)_{n}}{\left(q^{2} ; q^{2}\right)_{n}}
$$

is the generating function for partitions into distinct parts with even ample part size. Setting $y=1$ yielded the connection with the Rogers-Ramanujan identities. Setting $x=1, y=-1$ yields

$$
f_{o}(q)=\sum_{n \geqq 0} \frac{q^{n^{2}}}{(-q ; q)_{n}}=1+q-q^{2}+q^{3}-q^{6}+q^{7}+q^{9}-2 q^{10}+\cdots
$$

one of Ramanujan's fifth order mock theta functions (cf. [9]). Thus $f_{o}(q)$ is the generating function for the excess of the number of partitions of $n$ into distinct parts of even ample part size and an even upper parity index over those with an odd upper parity index. This is Theorem 17 as stated in the introduction.

For example, at $n=10$, the six partitions in question are $10,9+1,8+2,7+3$, $6+4,5+3+2$. Of these $9+1$ and $7+3$ have even upper parity index 0 while the other four have index 1.; thus the coefficient of $q^{10}$ in $f_{o}(q)$ must be $2-4=-2$.

In this section, we shall relate $\rho_{e}(\infty, y, a, q)$ to an identity involving the little $q$-Jacobi polynomials [9; p. 27].

\section{Theorem 18.}

$$
\begin{aligned}
\rho_{e}(\infty, y, a, q) & =\sum_{n \geqq 0} \frac{q^{n^{2}} a^{n}(-y q ; q)_{n}}{\left(q^{2} ; q^{2}\right)_{n}} \\
& =\frac{1}{(a q ; q)_{\infty}} \sum_{n \geqq 0} \frac{(-1)^{n} a^{n} q^{2 n^{2}}\left(a^{2} ; q^{2}\right)_{n}\left(1-a q^{2 n}\right)}{\left(q^{2} ; q^{2}\right)_{n}(1-a)} p_{n}\left(y ;-\frac{a}{q},-1: q\right),
\end{aligned}
$$

where the little q-Jacobi polynomial is given by [12; p. 27]

$$
p_{n}(y ; A, B: q)={ }_{2} \phi_{1}\left(\begin{array}{c}
q^{-n}, A B q^{n+1} ; q, q y \\
A q
\end{array}\right) .
$$

Proof. We recall the weak Bailey lemma [9; p. 116, eq. (2.5) with $\left.n, \rho_{1}, \rho_{2} \rightarrow \infty\right]$ (cf. $[9 ;$ p. 116, eq. (3.1) corrected])

$$
\sum_{n=0}^{\infty} q^{n^{2}} a^{n} \beta_{n}=\frac{1}{(a q ; q)_{\infty}} \sum_{n=0}^{\infty} q^{n^{2}} a^{n} \alpha_{n},
$$

where

$$
\beta_{n}=\sum_{r=0}^{n} \frac{\alpha_{r}}{(q ; q)_{n-r}(a q ; q)_{n+r}} .
$$


So if we take $\beta_{n}=(-y q ; q)_{n} /\left(q^{2} ; q^{2}\right)_{n}$, then by $[9 ;$ p. 115 , eq. $(2.7)]$

$$
\begin{aligned}
\alpha_{n} & =\left(1-a q^{2 n}\right) \sum_{j=0}^{n} \frac{(a q ; q)_{n+j-1}(-1)^{n-j} q^{\left(\begin{array}{c}
n-j \\
2
\end{array}\right)}(-y q ; q)_{j}}{(q ; q)_{n-j}\left(q^{2} ; q^{2}\right)_{j}} \\
& =\frac{(-1)^{n} q^{\left(\begin{array}{c}
n \\
2
\end{array}\right)}\left(1-a q^{2 n}\right)(a ; q)_{n}}{(1-a)(q ; q)_{n}}{ }_{3} \phi_{2}\left(\begin{array}{c}
q^{-n}, a q^{n},-y q ; q, q \\
-q, 0
\end{array}\right)
\end{aligned}
$$

(by $[12 ;$ p. 241 , eq. (III.7)])

$$
\begin{aligned}
& =\frac{(-1)^{n} q^{n^{2}}\left(a^{2} ; q^{2}\right)_{n}\left(1-a q^{2 n}\right)}{\left(q^{2} ; q^{2}\right)_{n}(1-a)}{ }_{2} \phi_{1}\left(\begin{array}{c}
q^{-n}, a q^{n} ; q, y q \\
-a
\end{array}\right) \\
& =(-1)^{n} q^{n^{2}}\left(a^{2} ; q^{2}\right)_{n}\left(1-a q^{2 n}\right) p_{n}\left(y ;-\frac{a}{q},-1: q\right),
\end{aligned}
$$

and substituting these values for $\alpha_{n}$ and $\beta_{n}$ into (12.5), we obtain the theorem.

Our first corollary is the classical identity of Rogers and Ramanujan [9; pp. 36-37, eq. (2.7.5) with $n \rightarrow \infty$ ] from which one deduces both Rogers-Ramanujan identities (the cases $a=1$ and $a=q$ ).

\section{Corollary 19.}

$$
\sum_{n=0}^{\infty} \frac{q^{n^{2}} a^{n}}{(q ; q)_{n}}=\frac{1}{(a q ; q)_{\infty}} \sum_{n=0}^{\infty} \frac{(a ; q)_{n}(-1)^{n} a^{n} q^{n(5 n-1) / 2}\left(1-a q^{2 n}\right)}{(q ; q)_{n}(1-a)} .
$$

Proof. Setting $y=1$, and noting by [12; p. 11, eq. (1.5.3)] that

$$
p_{n}(1 ; A, B: q)=\frac{\left(A B q^{n+1}\right)^{n}\left(B^{-1} q^{-n} ; q\right)_{n}}{(A q ; q)_{n}}=\frac{A^{n} q^{\left(\begin{array}{c}
n+1 \\
2
\end{array}\right)}(B q ; q)_{n}}{(A q ; q)_{n}},
$$

we find that (12.3) simplifies to (12.6).

In order to obtain the formula [9; p. 114, eq. (1.4)] for $f_{o}(q)$, we need some lemmas concerning the little $q$-Jacobi polynomials.

\section{Lemma 20.}

$$
p_{n}(-1 ;-1,-1: q)=(-1)^{n} q^{\left(\begin{array}{c}
n+1 \\
2
\end{array}\right)} \sum_{j=-n}^{n}(-1)^{j} q^{-j^{2}} .
$$

Proof. This result follows directly by mathematical induction provided we can show that

$$
(-1)^{n} q^{-\left(\begin{array}{c}
n+1 \\
2
\end{array}\right)} p_{n}(-1 ;-1,-1: q)-(-1)^{n-1} q^{-\left(\begin{array}{c}
n \\
2
\end{array}\right)} p_{n-1}(-1 ;-1,-1: q)=2(-1)^{n} q^{-n^{2}},
$$

or equivalently

$$
{ }_{2} \phi_{1}\left(\begin{array}{c}
q^{-n}, q^{n+1} ; q,-q \\
-q
\end{array}\right)+q^{n}{ }_{2} \phi_{1}\left(\begin{array}{c}
q^{-n+1}, q^{n} ; q,-q \\
-q
\end{array}\right)=2 q^{-\left(\begin{array}{c}
n \\
2
\end{array}\right) .}
$$


But

$$
\begin{aligned}
& { }_{2} \phi_{1}\left(\begin{array}{c}
q^{-n}, q^{n+1} ; q,-q \\
-q
\end{array}\right)+q_{2}^{n} \phi_{1}\left(\begin{array}{c}
q^{-n+1}, q^{n} ; q,-q \\
-q
\end{array}\right) \\
& =\sum_{j=0}^{n} \frac{\left(q^{-n+1} ; q\right)_{j-1}\left(q^{n+1} ; q\right)_{j-1}(-q)^{j}}{(q ; q)_{j}(-q ; q)_{j}}\left(\left(1-q^{-n}\right)\left(1-q^{n+j}\right)+q^{n}\left(1-q^{-n+j}\right)\left(1-q^{n}\right)\right) \\
& =\left(1+q^{n}\right)_{2} \phi_{1}\left(\begin{array}{c}
q^{-n}, q^{n} ; q,-q \\
-q
\end{array}\right) \\
& =2 q^{-\left(\begin{array}{c}
n \\
2
\end{array}\right)}
\end{aligned}
$$

by $[12 ;$ p. 11 , eq. (1.5.2)].

\section{Lemma 21.}

$$
2 p_{n}\left(-1 ;-\frac{1}{q},-1: q\right)-2 p_{n}\left(-1 ;-\frac{1}{q},-\frac{1}{q}: q\right)=\left(1-q^{n}\right) p_{n-1}(-1 ;-1,-1: q) .
$$

Proof.

$$
\begin{aligned}
2 p_{n}\left(-1 ;-\frac{1}{q},-1: q\right)-2 p_{n} & \left(-1 ;-\frac{1}{q},-\frac{1}{q}: q\right) \\
& =2 \phi_{1}\left(\begin{array}{c}
q^{-n}, q^{n} ; q,-q \\
-1
\end{array}\right)-2_{2} \phi_{1}\left(\begin{array}{c}
q^{-n}, q^{n-1} ; q,-q \\
-1
\end{array}\right) \\
& =2 \sum_{j=0}^{n} \frac{\left(q^{-n}, q\right)_{j}(-q)^{j}}{(q ; q)_{j}(-1 ; q)_{j}}\left(\left(1-q^{n+j-1}\right)-\left(1-q^{n-1}\right)\right) \\
& =2 \sum_{j=1}^{n} \frac{\left(q^{-n}, q\right)_{j}(-q)^{j}\left(q^{n}\right)_{j-1}}{(q ; q)_{j-1}(-1 ; q)_{j}} \\
& =\left(1-q^{n}\right)_{2} \phi_{1}\left(\begin{array}{c}
q^{-n+1}, q^{n} ; q,-q \\
-q
\end{array}\right) \\
& =\left(1-q^{n}\right) p_{n-1}(-1 ;-1,-1: q) .
\end{aligned}
$$

Lemma 22.

$$
2 p_{n}\left(-1 ;-\frac{1}{q},-1: q\right)=(-1)^{n} q^{\left(\begin{array}{c}
n+1 \\
2
\end{array}\right)} \sum_{j=-n}^{n}(-1)^{j} q^{-j^{2}}-(-1)^{n} q^{\left(\begin{array}{c}
n \\
2
\end{array}\right)} \sum_{j=-n+1}^{n-1}(-1)^{i} q^{-j^{2}}
$$

Proof. Noting that by $[12 ;$ p. 11 , eq. (1.5.2)]

$$
p_{n}\left(-1 ;-\frac{1}{q},-\frac{1}{q}: q\right)={ }_{2} \phi_{1}\left(\begin{array}{c}
q^{-n}, q^{n-1} ; q,-q \\
-1
\end{array}\right)=\frac{\left(-q^{1-n} ; q\right)_{n}}{(-1 ; q)_{n}}=q^{-\left(\begin{array}{c}
n \\
2
\end{array}\right)},
$$

we combine Lemmas 20 and 21 to see that

$$
\begin{aligned}
2 p_{n}\left(-1 ;-\frac{1}{q},-1: q\right) & =2 q^{-\left(\begin{array}{c}
n \\
2
\end{array}\right)}+\left(1-q^{n}\right)(-1)^{n-1} q^{\left(\begin{array}{c}
n \\
2
\end{array}\right)} \sum_{j=-n+1}^{n-1}(-1)^{j} q^{-j^{2}} \\
& =(-1)^{n} q^{\left(\begin{array}{c}
n+1 \\
2
\end{array}\right)} \sum_{j=-n}^{n}(-1)^{j} q^{-j^{2}}-(-1)^{n} q^{\left(\begin{array}{c}
n \\
2
\end{array}\right)} \sum_{j=-n+1}^{n-1}(-1)^{j} q^{-j^{2}},
\end{aligned}
$$

as desired. 
Corollary 23 ([9; p. 114, eq. (1.4)]).

$$
f_{0}(q)=\frac{1}{(q ; q)_{\infty}} \sum_{n=0}^{\infty} \sum_{|j| \leqq n}(-1)^{n} q^{n(5 n+1) / 2-j^{2}}\left(1-q^{4 n+2}\right) .
$$

Proof. Setting $y=-1, a=1$ in (12.3) and invoking Lemma 22, we see that

$$
\begin{aligned}
f_{0}(q) & =\sum_{n=0}^{\infty} \frac{q^{n^{2}}}{(-q ; q)_{n}} \\
& =\frac{1}{(q ; q)_{\infty}} \sum_{n=0}^{\infty} q^{n^{2}}(-1)^{n}\left((-1)^{n} q^{\left(\begin{array}{c}
n+1 \\
2
\end{array}\right)} \sum_{j=-n}^{n}(-1)^{j} q^{-j^{2}}-(-1)^{n} q^{\left(\begin{array}{c}
n \\
2
\end{array}\right)} \sum_{j=-n+1}^{n-1}(-1)^{j} q^{-j^{2}}\right) \\
& =\frac{1}{(q ; q)_{\infty}} \sum_{\substack{n=0 \\
|j| \leqq n}}^{\infty}(-1)^{j} q^{n(5 n+1) / 2-j^{2}}\left(1-q^{4 n+2}\right),
\end{aligned}
$$

where we have replaced $n$ by $n+1$ in the second double sum.

\section{Conclusion and Open Problems}

As was mentioned in Section 1 and again in Section 5, K. Kursungoz has found combinatorial proofs of many of the results in this paper. In particular, he has sieve-theoretic proofs of equations (7.4), (7.5), (8.4), (8.5) and (8.12); i.e. all the theorems in Sections 7 and 8. He has bijective proofs of (9.3), (9.4), (10.3), (10.4) and (11.2); i.e. all the theorems in Sections 9-11. Additionally, as was noted in Section 5 he has a combinatorial, bijective proof of Theorem 4 for all $k$, not just $k=2$ or 3 , and has beautifully generalized the result to cover all specializations to (1.6) with $1 \leqq a \leqq k$. On top of this he has bijective explanations of each of the multiple series generating functions given in Sections 3 and 4.

Furthermore, he has found a reasonably natural interpretation for

$$
\sum_{n_{1}, \ldots, n_{k-1} \geqq 0} \frac{q^{N_{1}^{2}+\cdots+N_{k-1}^{2}} x^{N_{1}+\cdots+N_{k-1}}(-y q ; q)_{n_{1}} \cdots(-y q ; q)_{n_{k-1}}}{\left(q^{2} ; q^{2}\right)_{n_{1}}\left(q^{2} ; q^{2}\right)_{n_{2}} \cdots\left(q^{2} ; q^{2}\right)_{n_{k-1}}} .
$$

The following are further problems that suggest themselves either from comparison of this work with results in the literature or from empirical studies.

1. Show bijectively that $\mathcal{W}_{3,3}(n)$ is equal to the number of partitions of $n$ into parts that differ by at least two and by more than 2 if the parts are even.

2. Show bijectively that $\mathcal{W}_{3,1}(n)$ is equal to the number of partitions of $n$ into parts $($ each $>2$ ) that differ by at least two and by more than 2 if the parts are even.

We know from Theorem 1 and a comparison of it with the Göllnitz-Gordon identities that in each case the sets of partitions involved are equinumerous. Thus the important word in each problem is "bijectively." More generally

3. Prove bijectively that

$$
\mathcal{W}_{2 k-1,2 a-1}(n)=D_{k, a}(n),
$$

where $D_{k, a}(n)$ denotes the number of partitions of $n$ of the form $n=\sum_{i \geqq 1} f_{i}-i$ (here we use the frequency notation $f_{i}$ for the appearances of $i$ in the partition) with $f_{1}+f_{2} \leqq a-1$ and for all $i \geqq 1$,

$$
f_{2 i-1} \leqq 1 \text { and } f_{2 i}+f_{2 i+1}+f_{2 i+2} \leqq k-1 .
$$


The assertion is true by comparing Theorem 1 here in the odd case with Theorem 1 in [2]. This problem and this observation leads naturally to a more general topic.

4. The overriding theme of this paper is parity. Can one generalize these theorems to moduli other than 2? In light of the fact that the above $D_{k, a}(n)$ is the special case $\lambda+1=2$ of the general partition theorem from [4] and [5], it would seem that theorems comparable to those in Sections 3-5 might be found by attempting to generalize the observation in Problem 3 to these more general results.

5. It follows from an old formula of Rogers [19; p. 333, eq. (6)] that

$$
P_{o}(-1,1 ; q)=\sum_{n=0}^{\infty} q^{n(3 n+1) / 2}\left(1-q^{2 n+1}\right) .
$$

Prove combinatorially that

$$
\sum_{r, m \geqq 0} p_{o}(r, m, N)(-1)^{r}= \begin{cases}1 & \text { if } N=n(3 n+1) / 2 \\ -1 & \text { if } N=n(3 n+5) / 2+1 \\ 0 & \text { otherwise }\end{cases}
$$

6. Prove combinatorially that $U_{o}(-1,-1 ; q)$ is equal to the third order mock theta function

$$
f(q)=1+\sum_{n>0} \frac{q^{n^{2}}}{\left((-q ; q)_{n}\right)^{2}} .
$$

In Problems 7 and 8 , let uc $(n)$ denote the coefficient of $q^{n}$ in the series for $(q, q)_{\infty} U_{o}(-1,-1 ; q)$.

7. Prove that $\mathrm{uc}(n)=8$ if $n$ is a prime congruent to 7 or $11 \bmod 12,-\mathrm{uc}(2)=$ $\mathrm{uc}(3)=\mathrm{uc}(5)=4$, and for all other primes $\mathrm{uc}(n)=0$.

8. Prove that uc $\left(n^{2}\right)=0$ if $n$ is an odd prime power and that $\mathrm{uc}\left(2^{n}\right)=-4$.

9. Prove that if $y=1$ and $x=-1$ in the second sum in (9.15) the result is

$$
\sum_{n=1}^{\infty}(-1)^{n} q^{n^{2}}
$$

10. Prove that if $y=1$ and $x=-1$ in the first sum in (9.16) the result is

$$
\sum_{n=1}^{\infty}(-1)^{n} q^{n^{2}}
$$

11. Extend the parity indices to overpartitions in a manner that will provide natural generalizations of the work of Corteel and Lovejoy [11], [18].

12. Provide a combinatorial proof of Lemma 20.

13. Noting that the theorems in Sections 9 and 10 are devoted to bi-basic generalizations of the $q$-binomial theorem and $q$-binomial series, find a full bi-basic generalization to bases other than $q$ and $q^{2}$.

14. An appealing, long-term project would be a full analytic proof of Theorem 4 and its extension to a more general $q$-hypergeometric series. We note that (5.1) can 
be rewritten as

$$
\begin{aligned}
& \sum_{\substack{n_{1}, \ldots, n_{k-1} \geqq 0 \\
j_{1}, \ldots j_{k-1} \geqq 0}} q^{\left(N_{1}+J_{1}\right)^{2}+\left(N_{2}+J_{2}\right)^{2}+\cdots+\left(N_{k-1}+J_{k-1}\right)^{2}} \\
& \quad \times \frac{x^{N_{1}+\cdots+N_{k-1}+J_{1}+\cdots J_{k-1}} y^{j_{1}+\cdots+j_{k-1}}}{\left(q^{2} ; q^{2}\right)_{n_{1}} \cdots\left(q^{2} ; q^{2}\right)_{n_{k-1}}\left(q^{2} ; q^{2}\right)_{j_{1}} \cdots\left(q^{2} ; q^{2}\right)_{j_{k-1}}} .
\end{aligned}
$$

where $N_{i}=n_{i}+n_{i+1}+\cdots+n_{k-1}$ and $J_{i}=j_{i}+j_{i+1}+\cdots+j_{k-1}$. This latter formulation seems likely to be a limiting case of series analogous to those considered in $[3]$.

15. Find a combinatorial interpretation for

$$
\left(-x q^{2} ; q^{2}\right)_{\infty} Q_{\frac{k}{2}, \frac{a}{2}}\left(x^{2} ; q^{2}\right)
$$

with $k$ even and $a$ odd. This is the "missing case" in Sections 3 and 4.

\section{ReFERENCES}

[1] G. E. Andrews, An analytic proof of the Rogers-Ramanujan-Gordon identities, Amer. J. Math., 88 (1966), 844-846.

[2] G. E. Andrews, A generalization of the Gölnitz-Gordon partition theorems, Proc. Amer. Math. Soc., 8 (1967), 945-952.

[3] G. E. Andrews, On q-difference equations for certain well-poised basic hypergeometric series, Quart. J. Math. Oxford Ser. (2), 19 (1968), 433-447.

[4] G. E. Andrews, A generalization of the classical partition theorems, Trans. Amer. Math. Soc., 145 (1968), 205-221.

[5] G. E. Andrews, On the general Rogers-Ramanujan theorem, Mem. Amer. Math. Soc., 152, (1974), 86 pp.

[6] G. E. Andrews, An analytic generalization of the Rogers-Ramanujan identities for odd moduli, Proc. Nat. Acad. Sci. USA, 71 (1974), 4082-4085.

[7] G. E. Andrews, The Theory of Partitions, Addison-Wesley, Reading, MA, 1976; reissued: Cambridge University Press, Cambridge, 1998.

[8] G. E. Andrews, Ramanujan's "lost" notebook, IV: stacks and alternating parity in partitions, Adv. in Math., 53 (1984), 55-74.

[9] G. E. Andrews, The fifth and seventh order mock theta functions, Trans. Amer. Math. Soc., 293 (1986), 113-134.

[10] G. E. Andrews and B. C. Berndt, Ramanujan's Lost Notebook, Part II Springer, New York, 2008.

[11] S. Corteel and J. Lovejoy, Overpartitions, Trans. Amer. Math. Soc., 356 (2004), 1623-1635.

[12] G. Gasper and M. Rahman, Basic Hypergeometric Series, Cambridge University Press, Cambridge, 1990.

[13] H. Göllnitz, Einfache Partionen, Diplomarbeit W.S. 1960, Göttingen, 65 pp.

[14] H. Göllnitz, Partitionen mit Differenzenbedingungen, J. Reine Angew. Math., 225 (1967), 154-190.

[15] B. Gordon, Some Ramanujan-like continued fractions, Abstracts of Short Communications, Int. Congr. of Math., Stockholm, 1962, pp. 29-30.

[16] B. Gordon, A combinatorial Generalization of the Rogers-Ramanujan identities, Amer. J. Math., 83 (1961), 393-399. 
[17] B. Gordon, Some continued fractions of the Rogers-Ramanujan type, Duke Math. J., 31 (1965), 741-748.

[18] J. Lovejoy, Overpartition Theorems of the Rogers-Ramanujan type, J. London Math. Soc. 69 (2004), 562-574.

[19] L. J. Rogers, On two theorems of combinatorial analysis and some allied identities, Proc. London Math. Soc. (2), 16 (1917), 315-336.

Department of Mathematics, The Pennsylvania State University, University Park, PA 16802

E-mail address: andrews@math.psu.edu 